\title{
NON-ABELIAN HODGE THEORY FOR ALGEBRAIC CURVES IN CHARACTERISTIC $p$
}

\author{
TSAO-HSIEN CHEN, XINWEN ZHU
}

\begin{abstract}
Let $G$ be a reductive group over an algebraically closed field of positive characteristic. Let $C$ be a smooth projective curve over $k$. We give a description of the moduli space of flat $G$-bundles in terms of the moduli space of $G$-Higgs bundles over the Frobenius twist $C^{\prime}$ of $C$. This description can be regarded as the non-abelian Hodge theory for curves in positive characteristic.
\end{abstract}

\section{Contents}

1. Introduction

2. Recollections of Hitchin fibrations

3. The non-abelian Hodge theory

Appendix A. The Stack of G-local systems

References

\section{INTRODUCTION}

Let $C$ be a Riemann surface, and $G_{c}$ be a compact Lie group with $G$ its complexification. Consider the triple $(E, \nabla, \phi)$, where $E$ is a $C^{\infty}$-principal $G_{c^{-}}$-bundle, $\nabla$ is a $C^{\infty}$-connection on $C$, and $\phi \in \operatorname{ad}(E) \otimes \mathcal{A}^{1,0}$ is a $(1,0)$ form on $C$ with values in the adjoint bundle $\operatorname{ad}(E)$. Then the famous Hitchin's equations are

$$
\left\{\begin{aligned}
F(\nabla)+\left[\phi, \phi^{*}\right] & =0, \\
\nabla^{\prime \prime}(\phi) & =0,
\end{aligned}\right.
$$

where $F(\nabla)$ is the curvature of $\nabla, \phi^{*}$ is the complex conjugation of $\phi$ (with respect to $G_{c}$ ), and $\nabla^{\prime \prime}$ is the $(0,1)$-component of the connection $\nabla$.

Hitchin obtained his equations by a dimensional reduction from $4 D$ to $2 D$ of the selfdual Yang-Mills equations, and found that the space of solutions of (1.1) has spectacular geometric properties. Namely, let $(E, \nabla, \phi)$ be a solution. Then $\nabla^{\prime \prime}$ endows $E \otimes \mathbb{C}$ with a structure as a holomorphic $G$-bundle, and $\phi$ as a holomorphic $\operatorname{ad}(E)$-valued 1-form, i.e $\phi \in \Gamma\left(C, \operatorname{ad}(E) \otimes \omega_{C}\right)$. Then the pair $(E, \phi)$ form a Higgs bundle. On the other hand, the connection $\tilde{\nabla}=\nabla+\left(\phi+\phi^{*}\right)$ is flat by (1.1). It is a well-known fact that a flat $C^{\infty}$-bundle on $C$ is the same as a holomorphic $G$-bundle with a flat connection, i.e., the pair $(E, \tilde{\nabla})$ forms a de Rham $G$-local system. Hitchin showed that a Higgs bundle $(E, \phi)$ on $C$ arises as a solution of (1.1) if and only it is (poly)stable with vanishing Chern class. Furthermore, the solution corresponding to $(E, \phi)$ is unique up to gauge transforms. In the other direction, Donaldson proved that every (semi)simple de Rham $G$-local system arises as a (unique up to gauge transforms) of the solution of (1.1). In this way, we get a bijection between stable $G$-Higgs bundles with vanishing Chern classes and irreducible $G$-local systems on $C$, which is furthermore a homeomorphism of the corresponding moduli spaces. This is what 
Simpson([S]) called the non-abelian Hodge theory for $C^{1}$. Note that the $(0,1)$-form $\phi^{*}$ plays a fundamental role as its presence changes the holomorphic structure on $E$.

It is been a while to search a version of this correspondence in characteristic $p$ case $([\mathrm{O}, \mathrm{OV}])$. However, compared with the story over $\mathbb{C}$, the picture is not very complete. In $[\mathrm{OV}]$, the Simpson correspondence is only established for connections with nilpotent $p$ curvatures.

In this note, we will establish a full version of the non-abelian Hodge theory for curves. For this purpose, let us first try to write down an analogue of the Hitchin's equations (1.1) in characteristic $p$. Assume that $G$ and $C$ are defined over an algebraically closed field $k$ of characteristic $p>0$. We assume that $p$ does not divide the order of the Weyl group of $G$. Let $F_{C}: C \rightarrow C^{\prime}$ be the relative Frobenius map, where $C^{\prime}$ is the pullback of $C$ along the absolute Frobenius of $k$. Let $(E, \tilde{\nabla})$ be a de Rham $G$-local system on $C$. In characteristic $p$, one can associate to $(E, \tilde{\nabla})$ its $p$-curvature, which is an $F$-Higgs field $\Psi(\tilde{\nabla}) \in \operatorname{ad}(E) \otimes F_{C}^{*} \omega_{C^{\prime}}$. Let $\nabla^{c a n}$ denote the canonical connection on $F_{C}^{*} \omega_{C^{\prime}}$. Let $\theta \in \operatorname{ad}(E) \otimes \omega_{C}$. The analogue of Hitchin's equations (1.1) in characteristic $p$ we have in mind are

$$
\left\{\begin{aligned}
\Psi(\tilde{\nabla}-\theta) & =0, \\
\left((\tilde{\nabla}-\theta) \otimes \nabla^{c a n}\right)(\Psi(\tilde{\nabla})) & =0
\end{aligned}\right.
$$

Let us explain the meaning of these equations. First note that there are some formal analogy between (1.1) and (1.2): The first equation is about the ( $p$-)curvature of a connection and the second equation is about the horizontality of some Lie algebra-valued one-from. The significance of these equations lies in the following observation. If we can find a solution $(E, \tilde{\nabla}, \theta)$ of $(1.2)$, then we can endow $E$ with a new flat connection $\nabla=\tilde{\nabla}-\theta$, whose $p$-curvature vanishes by the first equation of (1.2). Therefore, by the Cartier decent, $E$ with this new connection is a pullback of a $G$-torsor $E^{\prime}$ on $C^{\prime}$. The second equation in (1.2) implies that the $F$-Higgs field $\Psi(\tilde{\nabla})$ is horizontal with respect to $\nabla$ and therefore is a pull back of $\phi^{\prime} \in \operatorname{ad}\left(E^{\prime}\right) \otimes \omega_{C^{\prime}}$, again by the Cartier decent. In other words, a solution of (1.2) allows us to construct a Higgs bundle $\left(E^{\prime}, \phi^{\prime}\right)$ on $C^{\prime}$ from a de Rham local system $(E, \tilde{\nabla})$. Note that the role $\theta$ is similar to the role of $\phi^{*}$ in (1.1).

As is well-known, the $p$-curvature $\Psi(\tilde{\nabla})$ is horizontal with respect to the connection $\tilde{\nabla}$ (cf. $[\mathrm{K}, \S 5])$. Therefore, the second equation in (1.2) reduces to a matrix equation

$$
[\Psi(\tilde{\nabla}), \theta]=0 .
$$

On the other hand, the first equation (1.2) is an ODE of order $p-1$. Indeed, it is well-known that if we choose a coordinate $z$ on $C$, and let $A \in M_{n}(k[[z]])$ be a $k[[z]]$-valued $n \times n$ matrix, then the $p$-curvature of the connection $\partial_{z}+A$ can be represented as the matrix $\left(\partial_{z}+A\right)^{p-1} A$. Therefore, (1.2) in principle should be much simpler than (1.1) as the latter are non-linear PDEs.

Unfortunately, despite of its simple nature, we do not know how to find solutions of (1.2) directly. The work of $[\mathrm{OV}, \S 4.1]$ essentially shows that if $G=\mathrm{GL}_{n},(1.2)$ has solutions étale locally on $C$. The proof is not elementary and is based on the Azumaya property of the sheaf of crystalline operators. It seems that there is some difficulty to generalize this approach to groups other than $\mathrm{GL}_{n}$. The main problem, as we shall see, is that there is no canonical solution of (1.2). So it is not clear (to us) how to apply the Tannakian formalism here. In addition, the methods of loc. cit. seems not to give the information of the existence of solutions of $\theta$ over $C$. In fact, as we shall argue, over the whole curve $C$, one should consider another better-behaved system of equations, which has (1.2) as its local avatar.

Note that $(1.3)$ says that $\theta \in \operatorname{Lie} \operatorname{Aut}(E, \Psi(\tilde{\nabla}))$. The group scheme $\operatorname{Aut}(E, \Psi(\tilde{\nabla}))$ on $C$ is not well-behaved as the dimension of its fibers might jump. By the work of [DG, N1],

\footnotetext{
${ }^{1}$ And what other people called the Simpson correspondence.
} 
the $F$-Higgs bundle $(E, \Psi(\tilde{\nabla}))$ (i.e. a Higgs bundle valued in $\left.F^{*} \omega_{C^{\prime}}\right)$ defines a smooth commutative group scheme $J_{b^{p}}$ over $C$, where $b^{p}$ is the characteristic polynomial of $\Psi(\tilde{\nabla})$. This is the so-called regular centralizer of the Higgs field $(E, \Psi(\tilde{\nabla}))$, which canonically maps to $\operatorname{Aut}(E, \Psi(\tilde{\nabla}))$. Our first observation is that instead of imposing $\theta \in \operatorname{LieAut}(E, \Psi(\tilde{\nabla}))$, it is better to ask for $\theta \in \operatorname{Lie} J_{b^{p}}$. In other words, we replace the second equation of (1.2) by asking for a section $\theta \in$ Lie $J_{b^{p}}$. Our second observation is that the group scheme $J_{b^{p}}$ is the Frobenius pullback of a smooth group scheme $J_{b^{\prime}}^{\prime}$ on $C^{\prime}(\text { cf. Proposition } 3.1)^{2}$, and it makes

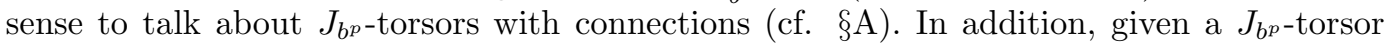
with a flat connection $\left(P, \nabla_{P}\right)$, there is a "product" flat connection $\nabla_{P \otimes E}$ on the $G$-torsor $P \otimes E:=P \times{ }^{J_{b p}} E$. Therefore, we may regard $\theta$ as a connection $d+\theta$ on $J_{b^{p}}$, and the first equation of (1.2) says that the $p$-curvature of the "product" connection on $E=J_{b^{p}} \otimes E$ vanishes. Finally, we replace $\left(J_{b^{p}}, d+\theta\right)$ by a general $J_{b^{p}}$-torsor with a connection $\left(P, \nabla_{P}\right)$ and consider the equation

$$
\Psi\left(\nabla_{P \otimes E}\right)=0 .
$$

As before, a solution $\left(E, \tilde{\nabla}, P, \nabla_{P}\right)$ of $(1.4)$ defines a Higgs bundle $\left(E^{\prime}, \phi^{\prime}\right)$ on $C^{\prime}$. Namely, since $\Psi(\tilde{\nabla})$ is preserved by $J_{b^{p}},(P \otimes E, \Psi(\tilde{\nabla}))$ is an $F$-Higgs bundle and (1.4) implies that it is a pullback of some $\left(E^{\prime}, \phi^{\prime}\right)$.

Note that such a quadruple $\left(E, \tilde{\nabla}, P, \nabla_{P}\right)$ is analogous to a harmonic bundle as introduced by Simpson $[\mathrm{S}]$. However, there is a fundamental difference. Namely, given $(E, \tilde{\nabla})$, there might be more than one $\left(P, \nabla_{P}\right)$ making $\left(E, \tilde{\nabla}, P, \nabla_{P}\right)$ a solution of (1.4). In fact, let $J_{b^{\prime}}^{\prime}$ be the smooth group scheme over $C^{\prime}$ mentioned above such that $J_{b^{p}}=F^{*} J_{b^{\prime}}^{\prime}$, and let $\mathscr{P}_{b^{\prime}}^{\prime}$ denote the Picard stack of $J_{b^{\prime}}^{\prime}$-torsors. Then the set of such $\left(P, \nabla_{P}\right)$, denoted by $\mathscr{H}_{b^{\prime}}^{-1}$, form a pseudo $\mathscr{P}_{b^{\prime}}^{\prime}$-torsor ${ }^{3}$ by tensoring $\left(P, \nabla_{P}\right)$ with the Frobenius pullback of a $J_{b^{\prime}}^{\prime}$-torsor. By considering all possible characteristic polynomials, we have a Picard stack $\mathscr{P}^{\prime}$ over the Hitchin base $B^{\prime}$ for the curve $C^{\prime}$ and a pseudo $\mathscr{P}^{\prime}$-torsor $\mathscr{H}^{-1}$ over $B^{\prime}$. It is convenient to consider its inverse, denoted by $\mathscr{H}$. In the main body of the paper, we will give another definition of $\mathscr{H}$ as an algebraic stack over $B^{\prime}$. Our first result is

Theorem 1.1. Under this action, the stack $\mathscr{H}$ form a $\mathscr{P}^{\prime}$-torsor.

The essentially point is to show that the map $\mathscr{H} \rightarrow B^{\prime}$ is surjective.

Now we state the main theorem of the note. Let Higgs ${ }_{G}^{\prime}$ denote the moduli space of Higgs bundles on $C^{\prime}$. According to [N1, N2], there is a canonical action of $\mathscr{P}^{\prime}$ on $\operatorname{Higgs}_{G}^{\prime}$. Let $\mathscr{H} \times \mathscr{P}^{\prime}$ Higgs $_{G}^{\prime}$ be the twist of $\operatorname{Higgs}_{G}^{\prime}$ by the $\mathscr{P}^{\prime}$-torsor $\mathscr{H}$.

Theorem 1.2. Over $B^{\prime}$, there is a canonical isomorphism

$$
\mathfrak{C}: \mathscr{H} \times{ }^{\mathscr{P}^{\prime}} \operatorname{Higgs}_{G}^{\prime} \rightarrow \operatorname{LocSys}_{G} .
$$

Roughly speaking, the map $\mathfrak{C}$ is inverse to the construction of a Higgs bundle from a solution of (1.4) as mentioned above.

Corollary 1.3. There is an étale cover $U$ of $B^{\prime}$ such that $\operatorname{LocSys}_{G} \times{ }_{B^{\prime}} U$ is isomorphic to $\operatorname{Higgs}_{G}^{\prime} \times{ }_{B^{\prime}} U$.

Proof. The Picard stack $\mathscr{P}^{\prime}$ is smooth over $B^{\prime}$, and therefore $\mathscr{H}$ can be trivialized étale locally on $B^{\prime}$.

Remark 1.4. In the case $G=\mathrm{GL}_{n}$, this corollary is one of the main theorems of [G], which extends a result of $[\mathrm{BB}]$ that establishes the above isomorphism away from the discriminant locus of $B^{\prime}$. However, the isomorphism $\mathfrak{C}$ was not obtained in loc. cit..

\footnotetext{
${ }^{2}$ But $F$-Higgs bundle $(E, \Psi(\tilde{\nabla}))$ is not the Frobenius pullback!

${ }^{3}$ Recall that a pseudo torsor is either an empty set or a torsor.
} 
Theorem 1.2 and Corollary 1.3 can be regarded as a non-abelian Hodge theory in characteristic $p$ as it says that $\operatorname{Higgs}_{G}^{\prime}$ and $\operatorname{LocSys}_{G}$ are isomorphic étale locally over $B^{\prime}$. Let us discuss some new features. First, our theorem is stated for all $G$-Higgs fields and all flat $G$-bundles, while over $\mathbb{C}$ such a correspondence could only be possible for (poly)stable Higgs bundles with vanishing Chern classes and (semi)simple flat $G$-local systems. Second, in characteristic $p$, what exists is the isomorphism $\mathfrak{C}$ rather than a direct isomorphism between $\operatorname{Higgs}_{G}^{\prime}$ and $\operatorname{LocSys}_{G}$. This is reminiscent of the transcendental nature of the Simpson correspondence over $\mathbb{C}$. Third, as the isomorphism $\mathfrak{C}$ is algebraic, one can compare directly between the tangent spaces of $\operatorname{Higgs}_{G}^{\prime}$ and $\operatorname{LocSys}_{G}$. In particular, one can obtain the comparison between Higgs cohomology and de Rham cohomology as a consequence of the global isomorphism $\mathfrak{C}$.

The relation between our work and the construction of $[\mathrm{OV}]$ (in the curve case) is as follows. The construction of $[\mathrm{OV}]$ amounts to the restriction of $\mathfrak{C}$ to $0 \in B^{\prime}$. Namely, upon a choice of a lifting of $C^{\prime}$ to $W_{2}(k),\left.\mathscr{H}\right|_{0}$ admits a canonical trivialization (see $\S 3.5$ ), and thus $\mathfrak{C}$ induces an isomorphism between the moduli of nilpotent $G$-Higgs bundles over $C^{\prime}$ and the moduli of flat $G$-bundles with nilpotent $p$-curvatures. We remark that it will be clear from our construction that if the group $G$ is bigger than $\mathrm{SL}_{2}$ there exist trivializations of $\left.\mathscr{H}\right|_{0}$ not induced from the lifting of $C^{\prime}$ to $W_{2}(k)$ (See Remark 3.24).

We also mention that the results in this note will play a fundamental role in our subsequent paper [CZ] to establish the (generic) geometric Langlands duality in prime characteristic. The main step is to identify the $\mathscr{P}^{\prime}$-torsor $\mathscr{H}$ with another $\mathscr{P}^{\prime}$-torsor over $B^{\prime}$ constructed from the Langlands dual group. We refer [CZ] for details.

Finally, it will be very interesting to find a generalization of these results to higher dimensional varieties. We just remark that Equation (1.2) makes sense for higher dimensional varieties. But solutions should only exist for those varieties that can be lifted to $W_{2}(k)$.

Let us briefly summarize the following sections. We will review the theory of Hitchin fibrations in $\S 2$, mostly following [N1, N2]. Readers familiar with this theory can skip most of it and go to $\S 2.3$ directly. Our main theorem is proved in $\S 3$. We construct the $p$-Hitchin map in $\S 3.1$, and then prove Theorem 1.1 in $\S 3.4$ by some cohomological argument. We briefly discuss the trivialization of the $\mathscr{P}_{0}^{\prime}$-torsor $\left.\mathscr{H}\right|_{0}$ is in $\S 3.5$. We establish our main theorem in §3.3. The proof is rather formal, after we develop some theories in Appendix A, which might be of independent interest. More precisely, we first develop the theory of de Rham $\mathcal{G}$-local systems for a non-constant group scheme $\mathcal{G}$ over $X$. The main observation is that the only additional input is a flat connection on $\mathcal{G}$ itself compatible with the group structure. Next, we discuss the $p$-curvature of such a de Rham $\mathcal{G}$-local system. We then develop the notion of the scheme of horizontal sections of a $\mathcal{D}_{X}$-scheme, which is the right adjoint of the pullback of $X^{\prime}$-schemes along the relative Frobenius. This is analogous to the same named notion in characteristic zero case developed in $([\mathrm{BD}, \S 2.6])$. We finish the note with a construction for every smooth commutative group scheme $\mathcal{G}^{\prime}$ over $X^{\prime}$, a 4 -term exact sequence of étale sheaves on $X^{\prime}$, which for $\mathcal{G}^{\prime}=\mathbb{G}_{m}$ specializes to a well-known exact sequence as in [I, §2.1].

We introduce the notations used throughout the note. We fix a smooth projective curve $C$ of genus at least two ${ }^{4}$ over an algebraically closed field $k$ of characteristic $p>0$. Let $\omega=\omega_{C}$ denote the canonical line bundle of $C$.

Let $S$ be a Noetherian scheme and $\mathscr{X} \rightarrow S$ be an algebraic stack over $S$. If $p \mathcal{O}_{S}=0$, we denote by $F r_{S}: S \rightarrow S$ be the absolute Frobenius map of $S$. We have the following

\footnotetext{
${ }^{4}$ This assumption should not be essential. We impose it to avoid the DG structure on moduli spaces.
} 
commutative diagram

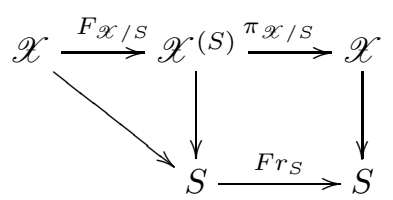

where the square is Cartesian. We call $\mathscr{X}^{(S)}$ the Frobenius twist of $\mathscr{X}$ along $S$, and $F_{\mathscr{X} / S}: \mathscr{X} \rightarrow \mathscr{X}^{(S)}$ the relative Frobenius morphism. If the base scheme $S$ is clear, $\mathscr{X}^{(S)}$ is also denoted by $\mathscr{X}^{\prime}$ for simplicity and $F_{\mathscr{X} / S}$ is denoted by $F_{\mathscr{X}}$ or $F$.

Let $\mathcal{G}$ be a smooth affine group scheme over $X$, and $E$ be a $\mathcal{G}$-torsor on $X$. We denote by $\operatorname{Aut}(E)=E \times^{\mathcal{G}} \mathcal{G}$ the adjoint torsor and $\operatorname{ad}(E)$ the adjoint bundle.

Let $G$ be a reductive algebraic group over $k$ of rank $l$. We denote by $\mathfrak{g}$ the Lie algebra of $G$. We assume that $p$ does not divide the order of the Weyl group W of $G$.

Acknowledgements. T-H. Chen would like to thank Roman Bezrukavnikov for many helpful discussions. We thank M. Groechenig for pointing out a mistake in an earlier version of the note. T-H. Chen is supported by NSF under the agreement No.DMS-1128155. X. Zhu is partially supported by NSF grant DMS-1001280/1313894 and DMS-1303296/1535464 and AMS Centennial Fellowship.

\section{Recollections of Hitchin fibRations}

In this section, we review some basic geometric facts of Hitchin fibrations, mostly following $[\mathrm{N} 1, \mathrm{~N} 2]$. Readers familiar with this theory can directly jump to the next section, probably except $§ 2.3$.

2.1. The Hitchin fibration. Let $k[\mathfrak{g}]$ and $k[\mathfrak{t}]$ be the algebra of polynomial function on $\mathfrak{g}$ and $\mathfrak{t}$. By Chevalley's theorem, we have an isomorphism $k[\mathfrak{g}]^{G} \simeq k[\mathfrak{t}]^{\mathrm{W}}$. Moreover, $k[\mathfrak{t}]^{\mathrm{W}}$ is isomorphic to a polynomial ring of $l$ variables $u_{1}, \ldots, u_{l}$ and each $u_{i}$ is homogeneous in degree $e_{i}$. Let $\mathfrak{c}=\operatorname{Spec}\left(k[\mathfrak{t}]^{W}\right)$. Let

$$
\chi: \mathfrak{g} \rightarrow \mathfrak{c}
$$

be the map induced by $k[\mathfrak{c}] \simeq k[\mathfrak{g}]^{G} \hookrightarrow k[\mathfrak{g}]$. This is $G \times \mathbb{G}_{m}$-equivariant map where $G$ acts trivially on $\mathfrak{c}$, and $\mathbb{G}_{m}$ acts on $\mathfrak{c}$ through the gradings on $k[\mathfrak{t}]^{\mathrm{W}}$. Let $\mathcal{L}$ be an invertible sheaf on $C$ and $\mathcal{L}^{\times}$be the corresponding $\mathbb{G}_{m}$-torsor. Let $\mathfrak{g}_{\mathcal{L}}=\mathfrak{g} \times{ }^{\mathbb{G}_{m}} \mathcal{L}^{\times}$and $\mathfrak{c}_{\mathcal{L}}=\mathfrak{c} \times{ }^{\mathbb{G}_{m}} \mathcal{L}^{\times}$be the $\mathbb{G}_{m}$-twist of $\mathfrak{g}$ and $\mathfrak{c}$ with respect to the natural $\mathbb{G}_{m}$-action.

Let $\operatorname{Higgs}_{G, \mathcal{L}}=\operatorname{Sect}\left(C,\left[\mathfrak{g}_{\mathcal{L}} / G\right]\right)$ be the stack of section of $\left[\mathfrak{g}_{\mathcal{L}} / G\right]$ over $C$, i.e., for each $k$-scheme $S$ the groupoid $\operatorname{Higgs}_{G, \mathcal{L}}(S)$ consists of maps over $C$ :

$$
h_{E, \phi}: C \times S \rightarrow\left[\mathfrak{g}_{\mathcal{L}} / G\right] .
$$

Equivalently, $\operatorname{Higgs}_{G, \mathcal{L}}(S)$ consists of a pair $(E, \phi)$ (called a Higgs bundle), where $E$ is a $G$-torsor over $C \times S$ and $\phi$ is an element in $\Gamma(C \times S, \operatorname{ad}(E) \otimes \mathcal{L})$. If the group $G$ is clear from the content, we simply write $\operatorname{Higgs}_{\mathcal{L}}$ for $\operatorname{Higgs}_{G, \mathcal{L}}$.

Let $B_{\mathcal{L}}=\operatorname{Sect}\left(C, \mathfrak{c}_{\mathcal{L}}\right)$ be the scheme of sections of $\mathfrak{c}_{\mathcal{L}}$ over $C$, i.e., for each $k$-scheme $S$, $B_{\mathcal{L}}(S)$ is the set of sections over $C$

$$
b: C \times S \rightarrow \mathfrak{c}_{\mathcal{L}} .
$$

This is called the Hitchin base of $G$.

The natural $G$-invariant projection $\chi: \mathfrak{g} \rightarrow \mathfrak{c}$ induces a map

$$
\left[\chi_{\mathcal{L}}\right]:\left[\mathfrak{g}_{\mathcal{L}} / G\right] \rightarrow \mathfrak{c}_{\mathcal{L}}
$$

which in turn induces a natural map

$$
h_{\mathcal{L}}: \operatorname{Higgs}_{\mathcal{L}}=\operatorname{Sect}\left(C,\left[\mathfrak{g}_{\mathcal{L}} / G\right]\right) \rightarrow \operatorname{Sect}\left(C, \mathfrak{c}_{\mathcal{L}}\right)=B_{\mathcal{L}} .
$$

We call $h_{\mathcal{L}}:$ Higgs $_{\mathcal{L}} \rightarrow B_{\mathcal{L}}$ the Hitchin map associated to $\mathcal{L}$. For any $b \in B_{\mathcal{L}}(S)$ we denote by Higgs ${ }_{\mathcal{L}, b}$ the fiber product $S \times{ }_{B_{\mathcal{L}}} \operatorname{Higgs}_{\mathcal{L}}$. 
We are mostly interested in the case $\mathcal{L}=\omega$. For simplicity, from now on we denote $B=B_{\omega}$, Higgs $=\operatorname{Higgs}_{\omega}, h=h_{\omega}$, and Higgs $\operatorname{Higgs}_{\omega_{C}}, b$, etc. We sometimes also write Higgs $_{G}$ for Higgs to emphasize the group $G$.

We fix a square root $\kappa=\omega^{1 / 2}$ (called a theta characteristic of $C$ ). Recall that in this case, there is a section $\epsilon_{\kappa}: B \rightarrow$ Higgs of $h$ : Higgs $\rightarrow B$, induced by the Kostant section $k o s: \mathfrak{c} \rightarrow \mathfrak{g}$. Sometimes, we also call $\epsilon_{\kappa}$ the Kostant section of the Hitchin fibration, and denote it by $\kappa$ for simplicity.

2.2. Symmetries of Hitchin fibration. Consider the group scheme $I$ over $\mathfrak{g}$ consisting of pairs

$$
I=\left\{(g, x) \in G \times \mathfrak{g} \mid \operatorname{Ad}_{g}(x)=x\right\} .
$$

The group scheme $I$ is not flat, but when restricted to the open subset of regular elements $\mathfrak{g}^{r e g}$, it is smooth. We define $J=k o s^{*} I$, This is called the universal centralizer group scheme of $\mathfrak{g}$, which is a smooth commutative group scheme over $\mathfrak{c}$. The following proposition is proved in [DG, N1]

Proposition 2.1. There is a canonical isomorphism of group schemes $\left.\left.\chi^{*} J\right|_{\mathfrak{g}^{\text {reg }}} \simeq I\right|_{\mathfrak{g}^{\text {reg }}}$, which extends to a morphism of group schemes $a: \chi^{*} J \rightarrow I \subset G \times \mathfrak{g}$.

All the above constructions can be twisted. Namely, there is a $\mathbb{G}_{m}$-action on $I$ given by $t \cdot(g, x)=(g, t x)$. It induced a $\mathbb{G}_{m}$-action on $J=k_{o s}^{*} I$, and the natural morphisms $J \rightarrow \mathfrak{c}$ and $I \rightarrow \mathfrak{g}$ are $\mathbb{G}_{m}$-equivariant. Therefore we can twist everything by a $\mathbb{G}_{m}$-torsor $\mathcal{L}^{\times}$and get $J_{\mathcal{L}} \rightarrow \mathfrak{c}_{\mathcal{L}}, I_{\mathcal{L}} \rightarrow \mathfrak{g}_{\mathcal{L}}$, where $J_{\mathcal{L}}=J \times{ }^{\mathbb{G}_{m}} \mathcal{L}^{\times}$and $I_{\mathcal{L}}=I \times{ }^{\mathbb{G}_{m}} \mathcal{L}^{\times}$. Moreover, there is a $G$-action on $I$ given by $h \cdot(g, x)=\left(h g h^{-1}, h x h^{-1}\right)$. The group scheme $I_{\mathcal{L}} \rightarrow \mathfrak{g}_{\mathcal{L}}$ is $G$-equivariant, and hence descends to a group scheme $\left[I_{\mathcal{L}}\right]$ over $\left[\mathfrak{g}_{\mathcal{L}} / G\right]$.

There is a natural action of the Picard stack $\mathscr{P}_{\mathcal{L}}$ of $J_{\mathcal{L}}$-torsors on Higgs ${ }_{\mathcal{L}}$. We recall its definition. Let $b: S \rightarrow B_{\mathcal{L}}$ be an $S$-point of $B_{\mathcal{L}}$, corresponding to a map $b: C \times S \rightarrow \mathfrak{c}_{\mathcal{L}}$. Pulling back $J_{\mathcal{L}} \rightarrow \mathfrak{c}_{\mathcal{L}}$ along this map defines a smooth group scheme $J_{b}=b^{*} J_{\mathcal{L}}$ over $C \times S$. Let $(E, \phi) \in \operatorname{Higgs}_{\mathcal{L}, b}$, corresponding to a map $h_{E, \phi}: C \times S \rightarrow\left[\mathfrak{g}_{\mathcal{L}} / G\right]$. Observe that the morphism $\chi^{*} J \rightarrow I$ in Proposition 2.1 induces $\left[\chi_{\mathcal{L}}\right]^{*} J_{\mathcal{L}} \rightarrow\left[I_{\mathcal{L}}\right]$ of group schemes over $\left[\mathfrak{g}_{\mathcal{L}} / G\right]$. Pulling back to $C \times S$ by $h_{E, \phi}$, we get a map

$$
a_{E, \phi}: J_{b} \rightarrow h_{E, \phi}^{*}\left[I_{\mathcal{L}}\right]=\operatorname{Aut}(E, \phi) \subset \operatorname{Aut}(E) .
$$

Therefore, we can twist $(E, \phi) \in \operatorname{Higgs}_{\mathcal{L}, b}$ by a $J_{b}$-torsor. This defines the promised action of $\mathscr{P}_{\mathcal{L}}$ on Higgs ${ }_{\mathcal{L}}$ over $B_{\mathcal{L}}$.

2.3. The tautological section $\tau: \mathfrak{c} \rightarrow$ Lie $J$. Recall that by Proposition 2.1, there is a canonical isomorphism $\left.\left.\chi^{*} J\right|_{\mathfrak{g}^{r e g}} \simeq I\right|_{\mathfrak{g}^{r e g}}$. The sheaf of Lie algebras Lie $\left(\left.I\right|_{\mathfrak{g}^{r e g}}\right) \subset \mathfrak{g}^{\text {reg }} \times \mathfrak{g}$ admits a tautological section given by $x \mapsto x \in$ Lie $I_{x}$ for $x \in \mathfrak{g}^{r e g}$. Clearly, this section descends to give a tautological section $\tau: \mathfrak{c} \rightarrow$ Lie $J$. We have the following property of $\tau$.

Lemma 2.2. Let $x \in \mathfrak{g}$, and $a_{x}: J_{\chi(x)} \rightarrow I_{x} \subset G$ be the homomorphism as in Proposition 2.1 (1). Then $d a_{x}(\tau \chi(x))=x$.

Proof. Consider the universal situation $x=\mathrm{id}: \mathfrak{g} \rightarrow \mathfrak{g}$. Then we need to show that $\chi^{*}(d a \circ \tau): \mathfrak{g} \rightarrow \chi^{*}$ Lie $J \rightarrow \mathfrak{g} \times \mathfrak{g}$ is the diagonal map. But by definition, this is true when restricted to $\mathfrak{g}^{\text {reg }} \subset \mathfrak{g}$. Therefore it holds over $\mathfrak{g}$.

Remark 2.3. In particular, if we take $x=0$, the lemma shows that $d a_{0}: \operatorname{Lie} J_{\chi(0)} \rightarrow \mathfrak{g}$ is not injective. I.e. the map $a: \chi^{*} J \rightarrow I$ is neither injective nor surjective over a general point $x \in \mathfrak{g}$.

Observe that $\mathbb{G}_{m}$ acts on $\mathfrak{g}^{r e g} \times \mathfrak{g}$ via natural homothethies on both factors, and therefore on $\chi^{*}$ Lie $\left.J\right|_{\mathfrak{g}^{r e g}} \simeq \operatorname{Lie}\left(\left.I\right|_{\mathfrak{g}^{r e g}}\right) \subset \mathfrak{g}^{\text {reg }} \times \mathfrak{g}$. This $\mathbb{G}_{m}$-action on $\chi^{*}$ Lie $\left.J\right|_{\mathfrak{g}^{r e g}}$ descends to a $\mathbb{G}_{m}$-action on Lie $J$ and for any line bundle $\mathcal{L}$ on $C$, the $\mathcal{L}^{\times}$-twist $(\operatorname{Lie} J) \times^{\mathbb{G}_{m}} \mathcal{L}^{\times}$under this $\mathbb{G}_{m}$ action is Lie $\left(J_{\mathcal{L}}\right) \otimes \mathcal{L}$, where $J_{\mathcal{L}}$ is introduced in $\S 2.2$. In addition, $\tau$ is $\mathbb{G}_{m}$-equivariant 
with respect to this $\mathbb{G}_{m}$ action on Lie $J$ and the natural $\mathbb{G}_{m}$ action on $\mathfrak{c}$. Therefore, if we define a vector bundle $B_{J, \mathcal{L}}$ over $B_{\mathcal{L}}$, whose fiber over $b \in B_{\mathcal{L}}$ is $\Gamma\left(C\right.$, Lie $\left.J_{b} \otimes \mathcal{L}\right)$, then twisting $\tau$ by $\mathcal{L}$, we obtain

$$
\tau_{\mathcal{L}}: B_{\mathcal{L}} \rightarrow B_{J, \mathcal{L}}
$$

which is a canonical section of the projection pr: $B_{J, \mathcal{L}} \rightarrow B_{\mathcal{L}}$.

\section{The NON-ABELIAN Hodge THEORY}

Let $\operatorname{LocSys}_{G}$ (or LocSys for brevity) be the moduli space of $G$-local systems on the curve $C$. The natural map $\operatorname{LocSys}_{G} \rightarrow \operatorname{Bun}_{G}$ can be regarded as a deformation of the map $\pi: T^{*} \operatorname{Bun}_{G} \rightarrow \operatorname{Bun}_{G}$. On the other hand, $T^{*} \operatorname{Bun}_{G}$ is just the moduli of Higgs field Higgs and there is the Hitchin fibration $h: \operatorname{Higgs}_{G} \rightarrow B$. In this section, we show that in the case char $k=p>0$, there is the $p$-Hitchin map $h_{p}: \operatorname{LocSys}_{G} \rightarrow B^{\prime}$, which is reminiscent of the classical Hitchin map in many aspects. In particular, we prove the non-abelian Hodge theory for $C$, which among other things, implies that étale locally on $B^{\prime}, h_{p}$ is isomorphic to $h^{\prime}: \operatorname{Higgs}_{G}^{\prime} \rightarrow B^{\prime}$. For general discussions of local systems on a smooth variety, we refer to Appendix A.

3.1. The $p$-Hitchin map for $G$-local systems. Let $\operatorname{LocSys}_{G}$ be the stack of $G$-local systems on $C$, i.e. for every scheme $S$ over $k, \operatorname{LocSys}_{G}(S)$ is the groupoid of all $G$-torsors $E$ on $C \times S$ together with a connection $\nabla: T_{C \times S / S} \rightarrow \widetilde{T}_{E}\left(\right.$ cf. $\S$ A.2). For every $(E, \nabla) \in \operatorname{LocSys}_{G}$ the $p$-curvature of $\nabla$ is an element $\Psi(\nabla) \in \Gamma\left(C, \operatorname{ad}(E) \otimes F_{C}^{*} \omega^{\prime}\right)$ (cf. $\left.\S A .6\right)$. Note that $F_{X}^{*} \omega^{\prime} \simeq \omega^{p}$, the $p$-th power of $\omega$. Thus we get an element $(E, \Psi(\nabla))$ in $\operatorname{Higgs}_{G, \omega^{p}}$, the stack of $F$-Higgs bundles, and the assignment $(E, \nabla) \rightarrow(E, \Psi(\nabla))$ defines a map

$$
\Psi_{G}: \operatorname{LocSys}_{G} \rightarrow \operatorname{Higgs}_{G, \omega^{p}} .
$$

Observe that pullback along $F_{C}: C \rightarrow C^{\prime}$ induces a natural map

$$
F^{p}: B^{\prime} \rightarrow B_{\omega^{p}} .
$$

In what follows, for $b^{\prime} \in B^{\prime}$, we denote by $b^{p}$ the image of $b^{\prime}$ under the map $F^{p}: B^{\prime} \rightarrow B_{\omega^{p}}$.

Proposition 3.1. There is a unique morphism $h_{p}: \operatorname{LocSys}_{G} \rightarrow B^{\prime}$, making the following diagram commute

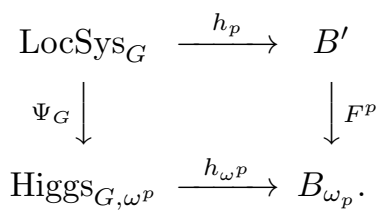

Proof. The argument is a variation of the proof of [LP, Proposition 3.2] which is due to J.B. Bost. Let $(E, \nabla) \in \operatorname{LocSys}_{G}$ and let $\Psi \in \Gamma\left(C, \operatorname{ad}(E) \otimes \omega^{p}\right)$ be the $p$-curvature of $\nabla$. As $\mathfrak{c}_{\omega^{p}}$ is the pullback of $\mathfrak{c}_{\omega}$ along $F_{C}: C \rightarrow C^{\prime}$, it has a canonical connection $\nabla^{\text {can }}$ and the scheme of horizontal sections $\mathfrak{c}_{\omega^{p}}^{\nabla^{\text {can }}}$ of $\mathfrak{c}_{\omega^{p}}$ is $\mathfrak{c}_{\omega^{\prime}}^{\prime}$ (see $\S$ A.7). Therefore, it is enough to show that the section $h_{\omega^{p}}(\Psi) \in \Gamma\left(C, \mathfrak{c}_{\omega^{p}}\right)$ is horizontal with respect to $\nabla^{c a n}$. This follows from the next lemma and the fact that the $p$-curvature $\Psi$ is horizontal with respect to the tensor connection on $\operatorname{ad}(E) \otimes \omega^{p}$.

Lemma 3.2. For a line bundle with a connection $\left(\mathcal{L}, \nabla_{\mathcal{L}}\right)$ on $C$ and a horizontal section $\phi \in \Gamma(C, \operatorname{ad}(E) \otimes \mathcal{L})$ (with respect to the tensor connection), the section $h_{\mathcal{L}}(E, \phi) \in \Gamma\left(C, \mathfrak{c}_{\mathcal{L}}\right)$ is a horizontal section of $\mathfrak{c}_{\mathcal{L}}$ (with respect to the connection induced from $\nabla_{\mathcal{L}}$ ).

Proof. This follows from the fact that $\operatorname{ad}(E) \otimes \mathcal{L} \rightarrow \mathfrak{c}_{\mathcal{L}}$ is a horizontal morphism of $\mathcal{D}_{C^{-}}$ schemes. 
We call $h_{p}$ the $p$-Hitchin fibration. Its fiber over $b^{\prime}$ is denoted by $\operatorname{LocSys}_{G, b^{\prime}}$.

Similarly to the usual Hitchin fibration, the $p$-Hitchin fibration has a large symmetry. We need a lemma. Let $(E, \nabla)$ be a $G$-local system on $C$ and $\Psi=\Psi(\nabla) \in \operatorname{ad}(E) \otimes \omega^{p}$ be the $p$-curvature. It induces a morphism $a_{E, \Psi}: J_{b^{p}} \rightarrow \operatorname{Aut}(E)$ as in $(2.1)$, where $b^{p}=h_{\omega^{p}}(E, \Psi)$ is equal to $F^{p}\left(b^{\prime}\right)$ for some $b^{\prime} \in B^{\prime}$. Observe that $J_{b^{p}}=F^{*} J_{b^{\prime}}^{\prime}$ is a $\mathcal{D}_{C^{-} \text {-group scheme so that }}$ $\left(J_{b^{p}}\right)^{\nabla}=J_{b^{\prime}}^{\prime}$. We refer to $\S \mathrm{A}$ for the detailed discussion of $\mathcal{D}_{C}$-group schemes.

Lemma 3.3. The morphism $a_{E, \Psi}$ is horizontal.

Proof. We consider a more general situation. Let $E \times \mathcal{L}^{\times}$be a $G \times \mathbb{G}_{m}$-local system on $C$. Observe that if $f: X \rightarrow Y$ is any $G \times \mathbb{G}_{m}$-equivariant morphism of schemes, then the twist of $f$ by $E \times \mathcal{L}^{\times}$, denoted by $f_{E \times \mathcal{L} \times}: X_{E \times \mathcal{L} \times} \rightarrow Y_{E \times \mathcal{L} \times}$ is a horizontal morphism of $\mathcal{D}_{C}$-schemes. If we apply the above construction to the following diagram

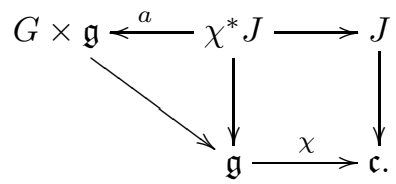

where $a: \chi^{*} J \rightarrow G \times \mathfrak{g}$ is as in Proposition 2.1 (1), we obtain a horizontal morphism $a_{E \times \mathcal{L} \times}: \chi_{E \times \mathcal{L} \times}^{*} J_{\mathcal{L}} \rightarrow \operatorname{Aut}(E)$ of group schemes over the total space of $\operatorname{ad}(E) \otimes \mathcal{L}$. If $\phi: C \rightarrow \operatorname{ad}(E) \otimes \mathcal{L}$ is a horizontal section, then $b_{\mathcal{L}}=h_{\mathcal{L}}(E, \phi) \in B_{\mathcal{L}}$ is a horizontal section of $\mathfrak{c}_{\mathcal{L}}$ by Lemma 3.2, and the pullback of $a_{E \times \mathcal{L} \times}$ along $\phi$ is $a_{E, \phi}: J_{b_{\mathcal{L}}} \rightarrow \operatorname{Aut}(E, \phi)$ as in (2.1), which is horizontal.

Finally, the lemma follows by applying the construction to $\mathcal{L}=\omega^{p}$ with the canonical connection, and $\phi=\Psi=\Psi(\nabla)$.

Note that the $\mathcal{D}_{C}$-group schemes $J_{b^{p}}$ can be organized into a family over $C \times B^{\prime}$, denoted by $J^{p} \rightarrow C \times B^{\prime}$. Namely, it is the pull back of the regular centralizers $J_{\omega^{\prime}}^{\prime} \rightarrow \mathfrak{c}_{\omega^{\prime}}^{\prime}$ along the map $C \times B^{\prime} \stackrel{F \times \text { id }}{\rightarrow} C^{\prime} \times B^{\prime} \rightarrow \mathfrak{c}_{\omega^{\prime}}^{\prime}$. Then $J^{p}$ is naturally a $\mathcal{D}_{C \times B^{\prime} / B^{\prime}}$-group scheme (cf. Example A.5) and for any $b^{\prime} \in B^{\prime}$, the restriction $\left.J^{p}\right|_{C \times\left\{b^{\prime}\right\}}$ is isomorphic to $J_{b^{p}}$. Therefore given $(P, \nabla) \in \operatorname{LocSys}_{J_{b p}}$, one can apply the formulation $\S$ A.5 to form a $G$-local system

$$
((P, \nabla),(E, \nabla)) \mapsto P \otimes E:=\left(a_{E, \Psi}\right)_{*} P \otimes E .
$$

It is easy to see that this induces a morphism $\otimes: \operatorname{LocSys}_{J^{p}} \times_{B^{\prime}} \operatorname{LocSys}_{G} \rightarrow \operatorname{LocSys}_{G}$.

Lemma 3.4. Assume that $h_{p}(E, \nabla)=b^{\prime}$. Let $P^{\prime}$ be a $J_{b^{\prime}}^{\prime}$-torsor, and $P=F^{*} P^{\prime}$ be the $J_{b^{p}}$-bundle with the canonical connection. Then $h_{p}\left(P \otimes E, \nabla_{P \otimes E}\right)=b^{\prime}$.

The question is local so one can assume that bundles are trivial. Then it is a direct calculation. We refer to Lemma 3.10 for more details, where a similar statement is proved. We identify $\mathscr{P}^{\prime}$ with the substack of $\operatorname{LocSys}_{J^{p}}$ with vanishing $p$-curvature. Then we obtain

Proposition 3.5. There is a natural action morphism $\mathscr{P}^{\prime} \times_{B^{\prime}} \operatorname{LocSys}_{G} \rightarrow \operatorname{LocSys}_{G}$ of stacks over $B^{\prime}$.

Remark 3.6. Alternatively, one can see the action of $\mathscr{P}^{\prime}$ on $\operatorname{LocSys}_{G}$ as follows. One can define a sheaf $\underline{\operatorname{Aut}}(E, \nabla)$ on $X_{e t}^{\prime}$ by assigning every étale map $f: U^{\prime} \rightarrow X^{\prime}$ the group

$$
\underline{\operatorname{Aut}}(E, \nabla)\left(U^{\prime}\right)=\operatorname{Aut}\left(f^{*} E, f^{*} \nabla\right) .
$$

One can show that there is an isomorphism $\operatorname{Aut}(E)^{\nabla}=\underline{\operatorname{Aut}}(E, \nabla)$ as étale sheaves on $X^{\prime}$. Then it follows from Lemma 3.3 that there is an action of $J_{b^{\prime}}^{\prime}$ on $(E, \nabla)$, and therefore we can twist $(E, \nabla)$ by a $J_{b^{\prime}}^{\prime}$-torsor. 
3.2. The stack $\mathscr{H}$. We will show that the $p$-Hitchin map $h_{p}:$ LocSys $\rightarrow B^{\prime}$ is closely related to the classical Hitchin map $h^{\prime}:$ Higgs $^{\prime} \rightarrow B^{\prime}$. Namely, they are related by a $\mathscr{P}^{\prime}$-torsor $\mathscr{H}$.

Let $b^{\prime} \in B^{\prime}$, and $J_{b^{\prime}}^{\prime}$ be the group scheme over $C^{\prime}$. Then $F_{C}^{*} J_{b^{\prime}}^{\prime}=J_{b^{p}}$ is a $\mathcal{D}_{C}$-group scheme. Therefore, it makes sense to define the stack LocSys $J_{b^{p}}$ of $J_{b^{p}}$-local systems on $C$, where $b^{\prime} \in B^{\prime}$. This is representable because the map LocSys $J_{b p}$ to the Picard stack $\mathscr{P}_{b^{p}}$ of $J_{b^{p}}$-torsors on $C$ is schematic. By Proposition 3.1 (or $\S$ A.8), there is the $p$-Hitchin map

$$
h_{J_{b^{\prime}}}: \operatorname{LocSys}_{J_{b p}} \rightarrow B_{J_{b^{\prime}}^{\prime}}^{\prime}:=\Gamma\left(C^{\prime}, \operatorname{Lie} J_{b^{\prime}} \otimes \omega_{C^{\prime}}\right) .
$$

Recall that $B_{J_{b^{\prime}}^{\prime}}^{\prime}$ is just the fiber of the vector bundle $B_{J^{\prime}, \omega^{\prime}}^{\prime}$ over $B^{\prime}$ introduced in $\S 2.3$. It is convenient to work with the universal situation, i.e. $b^{\prime}: B^{\prime} \rightarrow B^{\prime}$ is the identity map. Then the $p$-Hitchin map can be organized into a morphism

$$
h_{J^{\prime}}: \operatorname{LocSys}_{J^{p}} \rightarrow B_{J^{\prime}, \omega^{\prime}}^{\prime}
$$

over $B^{\prime}$. The result in A.9 implies

Lemma 3.7. This morphism is smooth.

Proof. First, both LocSys $J^{p}$ and $B_{J^{\prime}, \omega^{\prime}}^{\prime}$ are smooth Picard stacks over $B^{\prime}$. Namely, the latter is a vector bundle over $B^{\prime}$ and the former is an extension of the smooth Picard stack $\mathscr{P}_{\omega^{p}} \times_{B_{\omega^{p}}} B^{\prime}$ by a vector group. Therefore we can check the smoothness of $h_{J^{\prime}}$ fiberwise over $B^{\prime}$. In addition, the map $h_{J^{\prime}}$ is additive by (A.7). Now Lemma A.10 identifies the tangent map $d h_{J^{\prime}}$ at the unit with the edge map $c: E_{\infty}^{1} \rightarrow E_{2}^{0,1}$ in the "second spectral sequence" that calculating the hypercohomology of the de Rham complex $\left(\operatorname{Lie} J_{b^{p}} \rightarrow \operatorname{Lie} J_{b^{p}} \otimes \omega_{C}\right)$. But the latter is surjective because $E_{2}^{2,0} \simeq H^{2}\left(C^{\prime}\right.$, Lie $\left.J_{b^{\prime}}^{\prime}\right)=0$.

Recall the section $\tau^{\prime}: B^{\prime} \rightarrow B_{J^{\prime}, \omega^{\prime}}^{\prime}$ defined in (2.2). Let us define

$$
\mathscr{H}=\operatorname{LocSys}_{J^{p}}\left(\tau^{\prime}\right):=B^{\prime} \times_{B_{J^{\prime}, \omega^{\prime}}^{\prime}} \operatorname{LocSys}_{J^{p}},
$$

the base change of LocSys $J^{p} \rightarrow B_{J^{\prime}, \omega^{\prime}}^{\prime}$ along $\tau^{\prime}$. By Corollary A.6, this is a pseudo $\mathscr{P}^{\prime}-$ torsor. An object in $\mathscr{H}$ is a $J^{p}$-local system $(P, \nabla)$ with a specific $p$-curvature. We have the following theorem whose proof will be given in $\S 3.4$.

Theorem 3.8. The stack $\mathscr{H}$ is a $\mathscr{P}^{\prime}$-torsor.

3.3. The main theorem. We will prove our main theorem in this subsection, assuming Theorem 3.8 whose proof will be given in the next subsection.

We first construct

Proposition-Definition 3.9. There is a canonical morphism of stacks over $B^{\prime}$

$$
\mathfrak{C}: \mathscr{H} \times{ }^{\mathscr{P} \prime} \operatorname{Higgs}_{G}^{\prime} \rightarrow \operatorname{LocSys}_{G} .
$$

Proof. The construction of the morphism $\mathfrak{C}$ is given as follows. For any $\left(E^{\prime}, \phi^{\prime}\right) \in \operatorname{Higgs}_{b^{\prime}}^{\prime}$, there is a canonical morphism $a_{E^{\prime}, \phi^{\prime}}: J_{b^{\prime}}^{\prime} \rightarrow \operatorname{Aut}\left(E^{\prime}, \phi^{\prime}\right) \subset \operatorname{Aut}\left(E^{\prime}\right)(\operatorname{see}(2.1))$ and therefore via pullback there is a horizontal morphism of $\mathcal{D}_{C}$-groups

$$
F^{*} a_{E^{\prime}, \phi^{\prime}}=a_{F^{*} E^{\prime}, F^{*} \phi^{\prime}}: J_{b^{p}} \rightarrow \operatorname{Aut}\left(F^{*} E^{\prime}\right),
$$

where $F^{*} E^{\prime}$ is equipped with the canonical connection. Since $F^{*} E^{\prime}$ is naturally a $\operatorname{Aut}\left(F^{*} E^{\prime}\right) \times$ $G$-local system (see Example A.2), by the formalism of induction and tensor functors in $\S$ A.5, given $(P, \nabla) \in \operatorname{LocSys}_{J_{b}}$, one can form a $G$-local system

$$
\left((P, \nabla),\left(E^{\prime}, \phi^{\prime}\right)\right) \mapsto\left(F^{*} a_{E^{\prime}, \phi^{\prime}}\right)_{*} P \otimes F^{*} E^{\prime} .
$$

It is easy to see that this induces a morphism $\mathfrak{C}: \operatorname{LocSys}_{J^{p}} \times \mathscr{P P}^{\prime} \mathrm{Higgs}^{\prime} \rightarrow \operatorname{LocSys}_{G}$. Indeed, let $P^{\prime}$ be a $J_{b^{\prime}}^{\prime}$-torsor on $C^{\prime}$. By $(2.1)$, one can twist the Higgs field $\left(E^{\prime}, \phi^{\prime}\right)$ by $P^{\prime}$ and let $P^{\prime} \otimes\left(E^{\prime}, \phi^{\prime}\right)$ denote the new Higgs field. Then the claim amounts to

$$
\left(F^{*} a_{E^{\prime}, \phi^{\prime}}\right)_{*}\left(P \otimes F^{*} P^{\prime}\right) \otimes F^{*} E^{\prime}=\left(F^{*} a_{P^{\prime} \otimes\left(E^{\prime}, \phi^{\prime}\right)}\right)_{*} P \otimes F^{*} E^{\prime},
$$


which can be checked directly by definitions.

To show that $\mathfrak{C}$ is a morphism over $B^{\prime}$, it is enough to show the following lemma.

Lemma 3.10. If $\left(E^{\prime}, \phi^{\prime}\right) \in \operatorname{Higgs}_{b^{\prime}}^{\prime}$ and $(P, \nabla) \in \operatorname{LocSys}_{b^{p}}$ whose p-curvature is $\tau^{\prime}\left(b^{\prime}\right) \in$ $B_{J_{b^{\prime}}^{\prime}}^{\prime}$, then $h_{p}\left(\left(F^{*} a_{E^{\prime}, \phi^{\prime}}\right)_{*} P \otimes F^{*} E^{\prime}\right)=b^{\prime}$.

Proof. Let us regard $b^{\prime}: C^{\prime} \rightarrow \mathfrak{c}_{\omega}^{\prime}$ as a section. Then the question is local on $C$. So we can assume that $E^{\prime}$ and $P$ are trivialized, and denote the map $F^{*} a_{E^{\prime}, \phi^{\prime}}: J_{b^{p}} \rightarrow \operatorname{Aut}\left(F^{*} E^{\prime}\right)$ by $F^{*} a_{\phi^{\prime}}: J_{b^{p}} \rightarrow G \times C$. This is exactly the map $J_{b^{p}} \simeq I_{F^{*} \phi^{\prime}} \rightarrow G \times C$.

By definition, the $p$-curvature of the $G$-local system $F^{*} E^{\prime}=G \times C$ is zero, and the $p$ curvature of $\left(P, \nabla_{P}\right)$ is $\Psi\left(\nabla_{P}\right)=F^{*} \tau\left(b^{\prime}\right) \in \operatorname{Lie} J_{b^{p}} \otimes F^{*} \omega_{C^{\prime}}$. By the diagram (A.5), it is easy to see that the $p$-curvature of $\left(F^{*} a_{E^{\prime}, \phi^{\prime}}\right)_{*} P \otimes F^{*} E^{\prime}$ is given by the image of $\tau\left(b^{p}\right)=F^{*} \tau^{\prime}\left(b^{\prime}\right)$ under $d F^{*} a_{\phi^{\prime}}: \operatorname{Lie} J_{b^{p}} \otimes F^{*} \omega_{C^{\prime}} \rightarrow \mathfrak{g} \otimes F^{*} \omega_{C^{\prime}}$. But by $\S 2.3, d F^{*} a_{\phi^{\prime}}\left(\tau\left(b^{p}\right)\right)=F^{*} \phi^{\prime}$, and therefore its image under $\mathfrak{g}_{\omega^{p}} \rightarrow \mathfrak{c}_{\omega^{p}}$ is $b^{p}$. The lemma follows.

Remark 3.11. It is clear from the construction that $\mathfrak{C}$ is $\mathscr{P}^{\prime}$-equivariant.

Our main theorem is

Theorem 3.12. The morphism $\mathfrak{C}$ is an isomorphism.

Proof. To prove that $\mathfrak{C}$ is an isomorphism, we will construct the inverse morphism. This is essentially explained in the introduction: Given a $G$-local system $(E, \nabla)$, a solution of the equation (1.4) defines a Higgs bundle $\left(E^{\prime}, \phi^{\prime}\right)$. Here we make it precise. Namely, by Corollary A.6, the pseudo $\mathscr{P}^{\prime}$-torsor $\operatorname{LocSys}_{J^{p}}\left(-\tau^{\prime}\right)$ of $J^{p}$-local systems with $p$-curvature $-\tau^{\prime}$ is the inverse of $\mathscr{H}=\operatorname{LocSys}_{J^{p}}\left(\tau^{\prime}\right)$. In particular, it is a $\mathscr{P}^{\prime}$-torsor. Therefore, it is enough to construct a morphism

$$
\mathfrak{C}^{-1}: \mathscr{H}^{-1} \times \mathscr{P}^{\prime} \operatorname{LocSys}_{G} \rightarrow \operatorname{Higgs}_{G}^{\prime} .
$$

Let $\left(P, \nabla_{P}\right)$ be a $J_{b^{p}}$-local system with the $p$-curvature $-\tau\left(b^{p}\right)$ so that $\tilde{E}=\left(a_{E, \Psi}\right)_{*} P \otimes E$ is a $G$-local system. In addition, there is an F-Higgs field $\tilde{\Psi}$ on $\tilde{E}$. Indeed, $(\tilde{E}, \tilde{\Psi})$ is the twist of $(E, \Psi)$ by the underlying $J_{b^{p}}$-torsor $P$. In particular, under the classical Hitchin $\operatorname{map} h_{\omega^{p}}: \operatorname{Higgs}_{G, \omega^{p}} \rightarrow B_{\omega^{p}}, h_{\omega^{p}}(\tilde{E}, \tilde{\Psi})=b^{p}$.

We show that the connection on the $G$-local system $\tilde{E}$ has vanishing $p$-curvature and $\tilde{\Psi}$ is horizontal. Then $(\tilde{E}, \tilde{\Psi})=F^{*}\left(E^{\prime}, \psi^{\prime}\right)$, and $h^{\prime}\left(E^{\prime}, \psi^{\prime}\right)=b^{\prime}$. This construction provides the inverse map of $\mathfrak{C}$.

The question is local on $C$. We can trivialize $P, E$ and $\omega_{C}$. Then $\Psi \in \mathfrak{g} \otimes \mathcal{O}_{C}, \Psi_{P} \in \operatorname{Lie} J_{b^{p}}$, and by Lemma 2.2, $d a_{E, \Psi}: \operatorname{Lie} J_{b^{p}} \rightarrow \mathfrak{g} \otimes \mathcal{O}_{C}$ will send $\Psi_{P}$ to $-\Psi$.

Note that the $p$-curvature of $\tilde{E}$ with respect to its connection $\tilde{\nabla}$ (do not confuse with $\tilde{\Psi}$ ) is given by $\Psi_{P} \otimes 1+1 \otimes \Psi \in \operatorname{Der}_{\mathcal{O}_{C}}\left(\left(\mathcal{O}_{P} \otimes \mathcal{O}_{E}\right)^{J_{b} p}\right)$. Here we use the fact that $\Psi_{P} \otimes 1+1 \otimes \Psi$, which is a priori an element in $\operatorname{Der}_{\mathcal{O}_{C}}\left(\mathcal{O}_{P} \otimes \mathcal{O}_{E}\right)$, preserves $\left(\mathcal{O}_{P} \otimes \mathcal{O}_{E}\right)^{J_{b p}}$. It is clear that under the isomorphism $\left(\mathcal{O}_{P} \otimes \mathcal{O}_{E}\right)^{J_{b p}} \simeq \mathcal{O}_{E}$ induced by the trivialization, $\Psi_{P} \otimes 1$ maps to $d a_{E, \Psi}\left(\Psi_{P}\right)$. Therefore, $\Psi_{P} \otimes 1+1 \otimes \Psi=0$.

Finally, we show that $\tilde{\Psi}$ is horizontal. Again, the question is local and we pick up the trivialization of $P$. Then under the isomorphism $\tilde{E} \simeq E$ of $G$-bundles, $\tilde{\nabla}_{z}=\nabla_{z}+A$ for some $A \in \operatorname{Im}\left(a_{E, \Psi}: \operatorname{Lie} J_{b^{p}} \rightarrow \mathfrak{g} \otimes \mathcal{O}_{C}\right)$. Then the claim follows from $[A, \Psi]=0$ and $\nabla_{z}(\Psi)=0$.

Remark 3.13. It is instructive to look at the isomorphism $\mathfrak{C}$ in the case when $G=\mathrm{GL}_{n}$. For simplicity, we fix $b^{\prime} \in B^{\prime}$. We first describe the fibers $\operatorname{Higgs}_{G, b^{\prime}}^{\prime}, \operatorname{LocSys}_{G, b^{\prime}}$ and $\mathscr{H}_{b^{\prime}}$ in this case. 
Let $S_{b^{\prime}}^{\prime} \in T^{*} C^{\prime}$ be the spectral curve for $b^{\prime}$, and $S_{b^{p}} \subset T^{*} C^{\prime} \times_{C^{\prime}} C$ be the pullback of $S_{b^{\prime}}^{\prime}$ which fits into the following Cartesian diagram

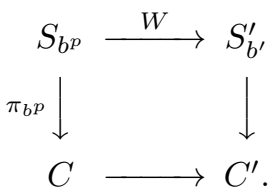

Note that $S_{b^{p}}$ is the spectral curve corresponding to $b^{p}=F^{*} b^{\prime}$.

Then Higgs $_{G, b^{\prime}}^{\prime}$ is the space of Higgs fields $\left(E^{\prime}, \phi^{\prime}\right)$ with the characteristic polynomial $b^{\prime}$. By the classical BNR correspondence (cf. [BNR]), such a Higgs field defines a coherent sheaf $\mathcal{F}_{\left(E^{\prime}, \phi^{\prime}\right)}$ on $S_{b^{\prime}}^{\prime}$. The space $\operatorname{LocSys}_{G, b^{\prime}}$ consist of rank $n$ vector bundles with a connection $(E, \nabla)$ on $C$ whose $p$-curvature $\Psi(\nabla)$ has the characteristic polynomial $b^{p}$. Therefore, every object in $\operatorname{LocSys}_{G, b^{\prime}}$ defines a coherent sheaf $\mathcal{F}_{(E, \Psi(\nabla))}$ on $S_{b^{p}}$. Finally,

$$
J_{b^{p}}=\pi_{b^{p}, *} \mathbb{G}_{m},
$$

and $\mathscr{H}_{b^{\prime}}$ can be regarded as the open substack of LocSys ${ }_{G, b^{\prime}}$ consisting of those $(E, \nabla)$, such that $\mathcal{L}:=\mathcal{F}_{(E, \Psi(\nabla))}$ is an invertible sheaf on $S_{b^{p}}$. Note that for such a pair $(E, \nabla) \in \mathscr{H}_{b^{\prime}}$, the direct image of $\mathcal{L}$ along $W: S_{b^{p}} \rightarrow S_{b^{\prime}}^{\prime}$ is locally free of rank $p$ on $S_{b^{\prime}}^{\prime}$ and therefore is a splitting module of the restriction to $S_{b^{\prime}}^{\prime}$ of the Azumaya algebra of the ring of crystalline differential operators $\mathcal{D}_{C}$. As a result, the map

$$
\mathfrak{C}_{b^{\prime}}: \mathscr{H}_{b^{\prime}} \times \mathscr{P}_{b^{\prime}}^{\prime} \operatorname{Higgs}_{G, b^{\prime}}^{\prime} \rightarrow \operatorname{LocSys}_{G, b^{\prime}}
$$

will send an object $\left(E^{\prime}, \phi^{\prime}\right) \in \operatorname{Higgs}_{G, b^{\prime}}^{\prime}$, regarded as a coherent sheaf $\mathcal{F}_{\left(E^{\prime}, \phi^{\prime}\right)}$ on $S_{b^{\prime}}^{\prime}$, and an object $\mathscr{H}_{b^{\prime}}$, which defines a splitting module $W_{*} \mathcal{L}$ of $\left.\mathcal{D}_{C}\right|_{S_{b^{\prime}}^{\prime}}$, to the tensor product $\mathcal{F}_{\left(E^{\prime}, \phi^{\prime}\right)} \otimes W_{*} \mathcal{L}$.

Remark 3.14. The description of $\mathscr{H}_{b^{\prime}}$ as a natural open substack of $\operatorname{LocSys}_{G, b^{\prime}}$ in the above example is a special feature for $G=\mathrm{GL}_{n}$. It is due to the existence of a natural open embedding $\mathscr{P}_{b^{\prime}}^{\prime}=\operatorname{Pic}\left(S_{b^{\prime}}^{\prime}\right) \subset \operatorname{Higgs}_{G, b^{\prime}}^{\prime}$.

In general, let $\operatorname{Higgs}_{G, \mathcal{L}}^{r e g}$ denote the open substack of $\operatorname{Higgs}_{G, \mathcal{L}}$ consisting of $(E, \phi): C \rightarrow$ $\left[\mathfrak{g}_{\mathcal{L}} / G\right]$ that factor through $C \rightarrow\left[\left(\mathfrak{g}^{\text {reg }}\right)_{\mathcal{L}} / G\right]$. It is known from [DG, N1] that $\operatorname{Higgs}_{\mathcal{L}}^{\text {reg }}$ is a $\mathscr{P}_{\mathcal{L}}$-torsor. Then a choice of the trivialization of this torsor (e.g. by a choice of Kostant section $\epsilon_{\mathcal{L}^{1 / 2}}$ ) defines an open embedding of $\mathscr{P}_{\mathcal{L}} \rightarrow \operatorname{Higgs}_{G, \mathcal{L}}^{\text {reg }}$.

Let us define an open substack $\operatorname{LocSys}_{G}^{r e g}$ of $\operatorname{LocSys}_{G}$ consisting of those $(E, \nabla)$ such that the $F$-Higgs field $(E, \Psi(\nabla)) \in \operatorname{Higgs}_{G, \omega^{p}}^{r e g}$. Then $\mathfrak{C}$ restricts to an isomorphism

$$
\mathscr{H} \times{ }^{\mathscr{P} \prime} \operatorname{Higgs}_{G}^{\prime r e g} \simeq \operatorname{LocSys}_{G}^{\text {reg }} .
$$

Therefore, a trivialization of Higgs ${ }_{G}^{\prime r e g}$ as the $\mathscr{P}^{\prime}$-torsor also defines an embedding $\mathscr{H} \simeq$ $\operatorname{LocSys}_{G}^{r e g} \subset \operatorname{LocSys}_{G}$. Note that as a corollary, we see that the map LocSys ${ }_{G}^{r e g} \rightarrow B^{\prime}$ is surjective, which is not obvious from its definition.

3.4. Proof of Theorem 3.8. This subsection is devoted to the proof of theorem 3.8.

By Lemma 3.7, $\mathscr{H}$ is smooth over $B^{\prime}$. So it is enough to show that $\mathscr{H} \rightarrow B^{\prime}$ is surjective. We will fix $b^{\prime} \in B^{\prime}(k)$. Let $\mathscr{G}_{\tau^{\prime}}$ be the $J^{\prime}$-gerbe corresponding to the section $\tau^{\prime}\left(b^{\prime}\right) \in$ $\Gamma\left(C^{\prime}\right.$, Lie $\left.J^{\prime} \otimes \omega_{C^{\prime}}\right)$ via the four term short exact sequence in Proposition A.7. Then $\mathscr{H}_{b^{\prime}}$ is just the pseudo Bun $J_{J^{\prime}}$-torsor of splittings of $\mathscr{G}_{\tau^{\prime}}$. We denote by $\left[b^{\prime}\right] \in H^{2}\left(C^{\prime}, J_{b^{\prime}}^{\prime}\right)$ the class corresponding to the gerbe $\mathscr{G}_{\tau_{b^{\prime}}^{\prime}}$. We need to show that $\left[b^{\prime}\right]$ is trivial. We begin with the following lemma.

Lemma 3.15. The class $\left[b^{\prime}\right]$ lies in the image of the map $H^{2}\left(C^{\prime},\left(J_{b^{\prime}}^{\prime}\right)^{0}\right) \rightarrow H^{2}\left(C^{\prime}, J_{b^{\prime}}^{\prime}\right)$. Here $\left(J_{b^{\prime}}^{\prime}\right)^{0}$ is the neutral component of $J_{b^{\prime}}^{\prime}$. 
Proof. By definition, the class $\left[b^{\prime}\right]$ is obtained from $\tau^{\prime}\left(b^{\prime}\right)$ via the exact sequence in Proposition A.7 applied to $J_{b^{\prime}}^{\prime}$. But we can also apply this sequence to $\left(J_{b^{\prime}}\right)^{0}$ to produce a class in $H^{2}\left(C^{\prime},\left(J_{b^{\prime}}^{\prime}\right)^{0}\right)$. Clearly, this new class will map to $\left[b^{\prime}\right]$.

Proposition 3.16. For any $b \in B(k)$ we have $H^{2}\left(C, J_{b}^{0}\right)=0$.

Clearly this proposition implies that $\left[b^{\prime}\right]$ is zero, and thus finish the proof of surjectivity of $\mathscr{H} \rightarrow B^{\prime}$. So it is enough to prove this proposition.

Proof. Let $K$ be the function field of $C$ and let $j: \eta=\operatorname{Spec} K \rightarrow C$ be the inclusion. Fix $b \in B(k)$ and we write $J_{\eta}^{0}:=\left.J_{b}^{0}\right|_{\eta}$. The group $J_{\eta}^{0}$ is smooth connected and commutative. Moreover, for a choice of a trivialization of $\omega_{C}^{\times}$at $\eta$ we have $J_{\eta}^{0} \simeq\left(G_{\eta}^{x}\right)^{0}$, where $x \in \mathfrak{g}^{\text {reg }}(K)$ is the image of $b \in \mathfrak{c}(K) \stackrel{k o s}{\rightarrow} \mathfrak{g}(K)$, and $\left(G_{\eta}^{x}\right)^{0}$ is the neutral component of the centralizer of $x$ in $G_{\eta}=G \times_{k} K$. There exists the largest affine subgroup $J_{\eta, s}^{0} \subset J_{\eta}^{0}$ of multiplicative type such that the quotient $U:=J_{\eta}^{0} / J_{\eta, s}^{0}$ is unipotent. Recall that a unipotent group over a field $K$ is called $K$-split if it admits a composition series with successive quotients $K$-isomorphic to $\mathbb{G}_{a}$.

Lemma 3.17. The unipotent group $U$ is $K$-split.

Proof. Let $F$ be the separable closure of $K$. By [CGP, Theorem B.3.4], it is enough to show that $U_{F}:=U \otimes_{K} F$ is $F$-split. We first construct a $\mathbb{G}_{m}$-action on $U_{F}$ defined over $F$. Let $x=x_{s}+x_{u}$ be the Jordan decomposition and $L_{\bar{\eta}}:=G_{\bar{\eta}}^{x_{s}}$ be the centralizer of $x_{s}$ in $G_{\bar{\eta}}=G \times_{k} \bar{F}^{5}$. It is known that $x_{u} \in \operatorname{Lie} L_{\bar{\eta}}$ is regular nilpotent and we have

$$
J_{\bar{\eta}}^{0}:=J_{F}^{0} \otimes_{F} \bar{F} \simeq\left(G_{\bar{\eta}}^{x}\right)^{0}=\left(L_{\bar{\eta}}^{x_{u}}\right)^{0} .
$$

There exists a co-character $2 \rho_{L}: \mathbb{G}_{m} \rightarrow L_{\bar{\eta}}$ such that $\operatorname{Ad}\left(2 \rho_{L}(t)\right)\left(x_{u}\right)=t^{2} x_{u}$. The cocharacter $2 \rho_{L}$ defines a $\mathbb{G}_{m}$-action $2 \rho_{L}\left(\mathbb{G}_{m}\right): \mathbb{G}_{m} \times J_{\bar{\eta}}^{0} \rightarrow J_{\bar{\eta}}^{0}$ on $J_{\bar{\eta}}^{0}$ by conjugation action. We claim that the action map $2 \rho_{L}\left(\mathbb{G}_{m}\right)$ is defined over $F$. Since the closed embedding $i: J_{\bar{\eta}}^{0} \hookrightarrow G_{\bar{\eta}}$ is defined over $F$, it is enough to show that the composition

$$
a: \mathbb{G}_{m} \times J_{\bar{\eta}}^{0} \rightarrow J_{\bar{\eta}}^{0} \hookrightarrow G_{\bar{\eta}}
$$

is defined over $F$. But in fact, $a$ factors as

$$
\mathbb{G}_{m} \times J_{\bar{\eta}}^{0} \stackrel{2 \rho_{L, G} \times i}{\longrightarrow} G_{\bar{\eta}} \times G_{\bar{\eta}} \stackrel{\mathrm{Ad}}{\rightarrow} G_{\bar{\eta}}
$$

where $2 \rho_{L, G}$ is the composition $2 \rho_{L, G}: \mathbb{G}_{m} \stackrel{2 \rho_{L}}{\rightarrow} L_{\bar{\eta}} \hookrightarrow G_{\bar{\eta}}$, which is defined over $F$ since it factors through a maximal torus of $G_{\bar{\eta}}$ and any maximal torus of $G_{\bar{\eta}}$ and any homomorphism between tori are defined over $F$. Since the adjoint action Ad of $G_{\bar{\eta}}$ is also defined over $F$, so is $a$. This finishes the construction of the promised $\mathbb{G}_{m}$-action on $J_{F}^{0}$ over $F$. Clearly, this $\mathbb{G}_{m}$-action preserves $J_{F, s}^{0}$ and therefore induces a $\mathbb{G}_{m}$-action on $U_{F} \simeq J_{F}^{0} / J_{F, s}^{0}$. By abuse of notation we still denote the action by $2 \rho_{L}\left(\mathbb{G}_{m}\right)$.

We next show that the action $2 \rho_{L}\left(\mathbb{G}_{m}\right)$ on $U_{F}$ has only nontrivial weights on Lie $U_{F}$. It is enough to prove this statement over $\bar{F}$. Let $B_{L}$ be the Borel subgroup of $L_{\bar{\eta}}$ such that $x_{u} \in$ Lie $U_{L}$, where $U_{L}$ is the unipotent radical of $B_{L}$. Without loss of generality, we can assume $x_{u}=\sum_{\alpha \in \Delta_{L}} x_{\alpha}$ and $2 \rho_{L}=\sum_{\alpha \in \Delta_{L}} \alpha^{\vee}$, where $\Delta_{L}$ is the set of simple roots of $L_{\bar{\eta}}$ determined by $B_{L}$ and $x_{\alpha}$ is a non-zero element in the corresponding root space. We have $U_{\bar{F}}=U_{L}^{x_{u}} \subset U_{L}$ and the statement follows from the fact that the action $2 \rho_{L}\left(\mathbb{G}_{m}\right)$ on $U_{\bar{F}}$ is the restriction of the conjugate action of $2 \rho_{L}$ on $U_{L}$ and the later action has only nontrivial weights on Lie $U_{L}$.

We have constructed an action of $\mathbb{G}_{m}$ on $U_{F}$ over $F$ with only nontrivial weights on Lie $U_{F}$. Then the lemma will follow from the general result.

\footnotetext{
${ }^{5}$ Notice that $x_{s}, x_{u} \in \mathfrak{g}(\bar{F})$ are not necessary in $\mathfrak{g}(F)$ since $F$ is not perfect.
} 
Lemma 3.18. Let $U$ be a smooth connected commutative affine unipotent group over a separably closed field $F$ of char $F=p>0$. If we have an action $\chi\left(\mathbb{G}_{m}\right)$ of $\mathbb{G}_{m}$ on $U$ over $F$ with only nontrivial weights on $\mathrm{Lie} U$, then $U$ is F-split.

Proof. Now by [CGP, Theorem B.3.4], there is a unique smooth connected $F$-split $F$ subgroup $U_{s}$ of $U$ such that $U_{w}:=U / U_{s}$ is $F$-wound (see loc. cit. for the definition of $F$-wound). We need to show that $U_{w}$ is trivial. By the uniqueness of $U_{s}$, the action $\chi\left(\mathbb{G}_{m}\right)$ preserves $U_{s}$ and induces a $\mathbb{G}_{m}$-action on $U_{w}$. On the other hand, by [CGP, Theorem B.4.3], any $\mathbb{G}_{m}$-action over $F$ on an $F$-wound smooth connected unipotent group is trivial. Thus, $\mathbb{G}_{m}$ acts trivially on $U_{w}$ and its Lie algebra Lie $U_{w}$ belongs to the zero weight space of Lie $U$. Therefore, by assumption. Lie $U_{w}=0$. Then $U_{w}$ is trivial since it is smooth and connected.

Lemma 3.19. We have $H^{i}\left(\operatorname{Spec} K, J_{\eta}^{0}\right)=0$ for $i \geq 1$.

Proof. We have an exact sequence $H^{i}\left(\operatorname{Spec} K, J_{\eta, s}^{0}\right) \rightarrow H^{i}\left(\operatorname{Spec} K, J_{\eta}^{0}\right) \rightarrow H^{i}(\operatorname{Spec} K, U)$. Thus it suffices to show that $H^{i}\left(\operatorname{Spec} K, J_{\eta, s}^{0}\right)=H^{i}(\operatorname{Spec} K, U)=0$ for $i \geq 1$. Since $\operatorname{dim} K \leq$ 1 and $J_{\eta, s}^{0}$ is a connected torus, we have $H^{i}\left(\operatorname{Spec} K, J_{\eta, s}^{0}\right)=0$ for $i \geq 1$. On the other hand, $U$ is $K$-split we have $H^{i}(\operatorname{Spec} K, U)=0$ for $i \geq 1$. We are done.

Finally, we prove Proposition 3.16. Since $C$ is a curve we have $H^{2}\left(C, J_{b}^{0}\right) \simeq H^{2}\left(\operatorname{Spec} K, j_{*} J_{\eta}^{0}\right)$. In addition, $R j_{*}^{i} J_{\eta}^{0}=0$ for $i \geq 1$. Indeed, the $i$-th direct image is the sheafification of the functor $S \rightarrow H^{i}\left(\operatorname{Spec} K \times_{C} S, J_{\eta}^{0}\right)$. Now $\operatorname{Spec} K \times_{C} S$ is the spectrum of a finite étale $K$ algebra, which is a finite product of finite separable extensions of $K$. Then the cohomology vanishes by Lemma 3.19. By Leray spectral sequence, this gives us

$$
H^{2}\left(C, J_{b}^{0}\right) \simeq H^{2}\left(\operatorname{Spec} K, j_{*} J_{\eta}^{0}\right) \simeq H^{2}\left(\operatorname{Spec} K, J_{\eta}^{0}\right),
$$

which vanishes by Lemma 3.19. This finishes the proof.

Example 3.20. Let us look at the most singular case $b=0 \in B(k)$. We assume that $G$ is semisimple for simplicity. Then $J_{b}=G^{e} \times^{\mathbb{G}_{m}} \omega_{C}^{\times}$, where $e \in \mathfrak{g}^{\text {reg }}$ is regular nilpotent. So the group $J_{\eta}^{0}$ is isomorphic to $J_{\eta}^{0} \simeq\left(G^{e}\right)^{0} \otimes_{k} K$. As the group $\left(G^{e}\right)^{0}$ is smooth connected and unipotent over an algebraically closed field $k, J_{\eta}^{0}$ is $K$-split.

3.5. Trivialization of $\mathscr{H}_{0}$. We briefly study trivializations of the $\mathscr{P}^{\prime}$-torsor $\mathscr{H}$ over $0 \in B^{\prime}$. We first deal the case $G=\mathrm{PGL}_{2}$, which is closely related to the geometry of the curve itself.

Lemma 3.21. If $G=\mathrm{PGL}_{2}$, then $J_{0}^{\prime} \simeq \omega_{C^{\prime}}^{-1}$, where $\omega_{C^{\prime}}^{-1}$ is regarded as an affine vector group over $C^{\prime}$.

As a result, $\mathscr{P}_{0}^{\prime} \simeq H^{1}\left(C^{\prime}, \omega_{C^{\prime}}^{-1}\right)$, and $\mathscr{H}_{0}$ is an $H^{1}\left(C^{\prime}, \omega_{C^{\prime}}^{-1}\right)$-torsor. In addition, for $b^{\prime}=0$, $J_{b^{p}}=F^{*} \omega_{C^{\prime}}^{-1}=\omega_{C}^{-p}$. So we have

Lemma 3.22. The stack $\mathscr{H}_{0}$ consists of $F^{*} \omega_{C^{\prime}}^{-1}$-torsors $E$ equipped with a connection $\nabla$, such that $h_{p}(E, \nabla)=1$ as an element in $\Gamma\left(C^{\prime}\right.$, Lie $\left.J_{0}^{\prime} \otimes \omega_{C^{\prime}}\right)=\Gamma\left(C^{\prime}, \omega_{C^{\prime}}^{-1} \otimes \omega_{C^{\prime}}\right)=\Gamma\left(C^{\prime}, \mathcal{O}_{C^{\prime}}\right)$.

Lemma 3.23. Under the above identification, the $\mathscr{P}_{0}^{\prime}$-torsor $\mathscr{H}_{0}$ is canonically isomorphic to the $H^{1}\left(C^{\prime}, \omega_{C^{\prime}}^{-1}\right)$-torsor of liftings of $C^{\prime}$ to $W_{2}(k)$.

Proof. This is essentially a reformulation of [OV, Theorem 4.5] in the curve case. Given a lifting $\widetilde{C}^{\prime}$ of $C^{\prime}$ to $W_{2}(k)$, we construct an object in $\mathscr{H}_{0}$ as follows. As explained in [OV, $\left.\S 1\right]$, a lifting of $C^{\prime}$ to $W_{2}(k)$ defines an extension of $\mathcal{O}_{C}$ by $F^{*} \omega_{C^{\prime}}^{-1}$ as $\mathcal{D}_{C^{-} \text {-modules }}$

$$
0 \rightarrow F^{*} \omega_{C^{\prime}}^{-1} \rightarrow \mathcal{E} \stackrel{\pi}{\rightarrow} \mathcal{O}_{C} \rightarrow 0 .
$$

such that the $p$-curvature of $\mathcal{E}$ is given by

$$
\mathcal{E} \rightarrow \mathcal{O}_{C} \simeq F^{*} \omega_{C^{\prime}}^{-1} \otimes F^{*} \omega_{C^{\prime}} \subset \mathcal{E} \otimes F^{*} \omega_{C^{\prime}}
$$


Then $\pi^{-1}(1)$ is an $F^{*} \omega_{C^{\prime}}^{-1}$-torsor on $C$, equipped with a connection ${ }^{6}$. By Lemma 3.22 , this defines an object of $\mathscr{H}_{0}$.

This construction induces a morphism from the $H^{1}\left(C^{\prime}, \omega_{C^{\prime}}^{-1}\right)$-torsor of liftings of $C^{\prime}$ to $W_{2}(k)$ to the $\mathscr{P}_{0}^{\prime}$-torsor $\mathscr{H}_{0}$, which clearly intertwines the action of $H^{1}\left(C^{\prime}, \omega_{C^{\prime}}^{-1}\right) \simeq \mathscr{P}_{0}^{\prime}$.

As a corollary, in the case $G=\mathrm{PGL}_{2}$ a choice of the lifting of $C^{\prime}$ to $W_{2}(k)$ gives rise to a trivialization of $\mathscr{H}_{0}$. The same is true for $G=\mathrm{SL}_{2}$, as $J_{0}^{\prime} \simeq \omega_{C^{\prime}}^{-1} \times \mu_{2}$. Now for a general reductive group $G$, we fix a principal $\varphi: \mathrm{SL}_{2} \rightarrow G$. Via pushout, we see that a lifting of $C^{\prime}$ to $W_{2}(k)$ gives rise to a trivialization of $\mathscr{H}_{0}$ as well.

Remark 3.24. If the group $G$ is bigger than $\mathrm{SL}_{2}$, then there are trivializations of $\mathscr{H}_{0}$ that do not come from liftings of $C^{\prime}$ to $W_{2}(k)$, as the dimension of $\mathscr{H}_{0}$ is bigger than the dimension of the $H^{1}\left(C^{\prime}, \omega_{C^{\prime}}^{-1}\right)$-torsor of liftings of $C^{\prime}$ to $W_{2}(k)$. Therefore, for group $G$ bigger than $\mathrm{SL}_{2}$, there are some Simpson correspondences that do not arise from the construction in $[\mathrm{OV}]$.

\section{Appendix A. The Stack of $\mathcal{G}$-Local systems}

In this appendix we discuss the notion of (de Rham) $\mathcal{G}$-local systems and their $p$-curvatures. We will fix a smooth morphism $X \rightarrow S$ of noetherian schemes (however all discussions carry through without change if $X$ is a smooth Deligne-Mumford stack), and a smooth affine group scheme $\mathcal{G}$ over $X$. We do not assume that $\mathcal{G}$ is constant or is fiberwise connected. The main example is the regular centralizer group scheme $J_{b^{p}}$ as in the note.

For any scheme $X \rightarrow S$ smooth over $S$, we denote by $T_{X / S}$ (resp. $\Omega_{X / S}$ ) its tangent (resp. cotangent) sheaf relative to $S$, or sometimes by $T_{X}$ (resp. $\Omega_{X}$ ) if no confusion will likely arise. We denote by $\mathcal{D}_{X / S}$ (or by $\mathcal{D}_{X}$ for simplicity) the sheaf of crystalline differential operators on $X$ as in [BB, OV].

A.1. Connections on $\mathcal{G}$-torsors. In order to talk about a connection on a $\mathcal{G}$-torsor, we need to assume that $\mathcal{G}$ itself is a $\mathcal{D}_{X / S}$-group scheme. I.e. it is equipped with a flat connection

$$
\nabla_{\mathcal{G}}: \mathcal{O}_{\mathcal{G}} \rightarrow \mathcal{O}_{\mathcal{G}} \otimes_{\mathcal{O}_{X}} \Omega_{X / S}
$$

which is compatible with the unit, the multiplication and the co-multiplication on $\mathcal{O}_{g}$. Equivalently, let $\Delta: X \rightarrow X \times_{S} X$ be the diagonal embedding and let $\Delta^{(1)}$ be the first infinitesimal neighborhood of $\Delta$ with two natural projections $p_{1}, p_{2}: \Delta^{(1)} \rightarrow X$. Then a flat connection on $\mathcal{G}$ is an isomorphism of group schemes $\nabla_{\mathcal{G}}: p_{1}^{*}(\mathcal{G}) \simeq p_{2}^{*}(\mathcal{G})$ that restricts to the identity map on $\Delta$ and that satisfies the usual cocycle condition when pull back to the first infinitesimal neighborhood $\Delta_{3}^{(1)}$ of the main diagonal $\Delta_{3}: X \rightarrow X \times X \times X$.

Given a $\mathcal{G}$-torsor $E$, a flat connection on $E$ is a $\mathcal{D}_{X / S}$-scheme structure on $E$ that is compatible with the $\mathcal{G}$-action. Explicitly, it is a flat connection $\nabla: \mathcal{O}_{E} \rightarrow \mathcal{O}_{E} \otimes_{\mathcal{O}_{X}} \Omega_{X / S}$, which is compatible with the multiplication of $\mathcal{O}_{E}$ and fits into the following commutative diagram

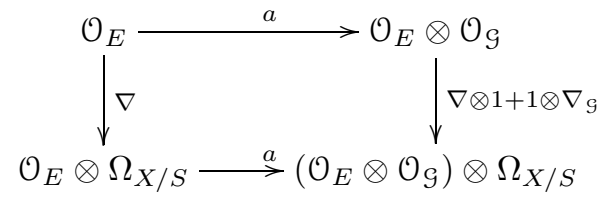

where $a: \mathcal{O}_{E} \rightarrow \mathcal{O}_{E} \otimes \mathcal{O}_{\mathcal{G}}$ is the co-action map. Equivalently, a flat connection on $E$ is an isomorphism $\nabla: p_{1}^{*}(E) \simeq p_{2}^{*}(E)$ of $p_{1}^{*} \mathcal{G} \simeq p_{2}^{*} \mathcal{G}$-torsors on $\Delta^{(1)}$ that restricts to the identity on $\Delta$ and that satisfies the usual cocycle condition on $\Delta_{3}^{(1)}$.

We denote by $\operatorname{LocSys}_{\mathcal{G}}$ the stack of $\mathcal{G}$-torsors with flat connections (or $\mathcal{G}$-local systems). Note that the following discussions do not require the representability of this stack.

\footnotetext{
${ }^{6}$ This is in fact the torsor of liftings of the Frobenius $F: C \rightarrow C^{\prime}$ to $W_{2}(k)$.
} 
Example A.1. In the case of constant group scheme $\mathcal{G}=G \times_{S} X$, there is a canonical connection on $\mathcal{O}_{\mathcal{G}}=\mathcal{O}_{G} \otimes_{\mathcal{O}_{S}} \mathcal{O}_{X}$ coming from $\mathcal{O}_{X}$. The above definition then reduces to the standard one.

A.2. Lie algebroid definition. Here is an equivalence definition. Let $\mathcal{G}$ be a smooth affine $\mathcal{D}_{X / S}$-group scheme as before. Let $E$ be a $\mathcal{G}$-torsor. Let us denote by $\widetilde{T}_{E}$ the Lie algebroid of infinitesimal symmetry of $E$ : a section of $\widetilde{T}_{E}$ is a pair $(v, \tilde{v})$, where $v \in T_{X / S}$ and $\tilde{v} \in T_{E}$ is a vector field on $E$ such that:

(1) The restriction on $\tilde{v}$ to $\mathcal{O}_{X} \subset \mathcal{O}_{E}$ is equal to $v$ (i.e. $\tilde{v}$ is a lifting of $v$ ).

(2) $\tilde{v}$ is $\mathcal{G}$-invariant, i.e. the following diagram commutes

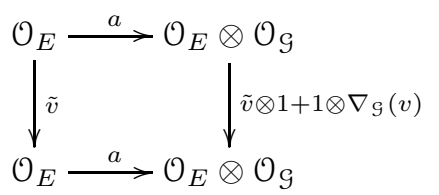

Let $\sigma: \widetilde{T}_{E} \rightarrow T_{X / S}$ be the projection map $(v, \tilde{v}) \rightarrow v$. There is the following exact sequence

$$
0 \rightarrow \operatorname{ad}(E) \rightarrow \widetilde{T}_{E} \stackrel{\sigma}{\rightarrow} T_{X / S}
$$

A connection $\nabla$ on $E$ is a splitting of this exact sequence, i.e. $\nabla$ is a map $\nabla: T_{X / S} \rightarrow \widetilde{T}_{E}$ such that $\sigma \circ \nabla=$ id. If in addition $\nabla$ is a Lie algebroid homomorphism, we say that $\nabla$ is a flat connection.

A.3. Connections on the trivial $\mathcal{G}$-torsor. Let $E=E^{0}$ be the trivial $\mathcal{G}$-torsor. Then it is equipped with a canonical flat connection coming from $\nabla_{\mathcal{G}}$, denoted by $\nabla^{0}$. Then by $\S$ A.2, the sheaf of connections on $E^{0}$ is isomorphic to Lie $\mathcal{9} \otimes \Omega_{X / S}$ via $\nabla \mapsto \nabla-\nabla^{0}$. We denote the subsheaf of flat connections on $E^{0}$ by $\left(\mathrm{Lie} \mathcal{G} \otimes \Omega_{X / S}\right)^{c l}$. If $\mathcal{G}=G \times X$ is constant, then $\left(\operatorname{Lie} \mathcal{G} \otimes \Omega_{X / S}\right)^{c l}=\operatorname{Lie} G \otimes z_{X / S}$, where $z_{X / S} \subset \Omega_{X / S}$ is the sheaf of closed one-forms.

There is always the following map of sheaves on $X$

$$
d \log : \mathcal{G} \rightarrow\left(\operatorname{Lie} \mathcal{G} \otimes \Omega_{X / S}\right)^{c l},
$$

defined as follows. We regard $g \in \mathcal{G}$ as an element in $\mathcal{H o m}_{\mathcal{O}_{X}}\left(\mathcal{O}_{\mathcal{G}}, \mathcal{O}_{X}\right)$, which carries on a natural connection, still denoted by $\nabla_{\mathcal{G}}$. Let $\mathcal{J}=\operatorname{ker} g \subset \mathcal{O}_{\mathcal{G}}$. Then it is easy to see that $\nabla_{\mathcal{G}}(g) \in \mathcal{H} \operatorname{Hom}\left(\mathcal{O}_{\mathcal{G}}, \mathcal{O}\right) \otimes \Omega_{X / S}$ annihilates $\mathcal{J}_{g}^{2}$, and therefore induces a map

$$
\nabla_{\mathcal{G}}(g): \mathcal{J}_{g} / \mathcal{J}_{g}^{2} \rightarrow \Omega_{X / S}
$$

Then $g^{-1} \nabla_{\mathcal{G}}(g)$ can be regarded as an element in Lie $\mathcal{G} \otimes \Omega_{X / S}$, and $\nabla^{0}+d \log (g)$ defines a connection on $E^{0}$. Note that $\mathcal{G}$ acts on $E^{0}$, and therefore on $\tilde{T}_{E^{0}}$. It is easy to see that $g:\left(E^{0}, \nabla^{0}+d \log (g)\right) \simeq\left(E^{0}, \nabla^{0}\right)$ is an isomorphism. In particular, $\nabla^{0}+d \log (g)$ is a flat connection, i.e. $d \log (g) \in\left(\operatorname{Lie} \mathcal{G} \otimes \Omega_{X / S}\right)^{c l}$.

A.4. Bitorsors and connection on bitorsors. Let $\mathcal{G}_{1}$ and $\mathcal{G}_{2}$ be two smooth affine group schemes. A $\left(\mathcal{G}_{1} \times \mathcal{G}_{2}\right)$-bitorsor on $X$ is a scheme $E$ on $X$ with a $\left(\mathcal{G}_{1} \times \mathcal{G}_{2}\right)$-action that makes $E$ into a left $\mathcal{G}_{1}$-torsor and a right $\mathcal{G}_{2}$-torsor. If $\mathcal{G}_{1}$ and $\mathcal{G}_{2}$ are $\mathcal{D}_{X / S}$ group schemes, we can similarly define the notion of a flat connection on a $\left(\mathcal{G}_{1} \times \mathcal{G}_{2}\right)$-bitorsor $E$, i.e. an isomorphism $\nabla: p_{1}^{*}(E) \simeq p_{2}^{*}(E)$ of bitorsors satisfying the usual conditions as before.

We denote by LocSys $_{\mathcal{G}_{1} \times \mathcal{G}_{2}}$ the stack of $\left(\mathcal{G}_{1} \times \mathcal{G}_{2}\right)$-bitorsors with flat connections.

Example A.2. Let $\mathcal{G}$ be a smooth affine $\mathcal{D}_{X / S^{-}}$group scheme over $X$ and let $E \in$ LocSys $_{\mathcal{G}}$. Let $\operatorname{Aut}(E)$ be the group scheme of automorphisms of $E$ (as a $\mathcal{G}$-torsor). Then $E$ has a natural $(\operatorname{Aut}(E) \times \mathcal{G})$-bitorsor structure. In addition, the group scheme $\operatorname{Aut}(E)$ has a canonical flat connection and $E \in \operatorname{LocSys}_{A u t(E) \times G}$. To see this, observe that $\operatorname{Aut}(E) \simeq$ $E \times{ }^{\mathcal{G}} \mathcal{G}$ and for any $Y \rightarrow X$ we have $\operatorname{Aut}(E)_{Y} \simeq E_{Y} \times \mathcal{G}_{Y} \mathcal{G}_{Y}$. Thus, the connections on $E$ 
and on $\mathcal{G}$ induce an isomorphism $p_{1}^{*} \operatorname{Aut}(E) \simeq p_{2}^{*} \operatorname{Aut}(E)$ on $\Delta^{(1)}$, which defines a connection on $\operatorname{Aut}(E)$. It is clear from the above construction that $E \in \operatorname{LocSys}_{\operatorname{Aut}(E) \times \mathcal{G}}$.

A.5. Some functors. Let $f: Y \rightarrow X$ be morphism between smooth schemes and let $\mathcal{G}_{X}$ be a smooth affine $\mathcal{D}_{X / S^{-}}$group scheme on $X$. Let $\mathcal{G}_{Y}:=f^{*} \mathcal{G}_{X}$. Then $\mathcal{G}_{Y}$ is a $\mathcal{D}_{Y / S^{-}}$group scheme and the pullback of $f$ defines a functor

$$
f^{*}: \operatorname{LocSys}_{\mathcal{G}_{X}} \rightarrow \operatorname{LocSys}_{\mathcal{G}_{Y}},
$$

sometimes called the pullback functor.

Assume that $\mathcal{G}_{1}$ and $\mathcal{G}_{2}$ are two smooth affine $\mathcal{D}_{X / S}$-group schemes and $h: \mathcal{G}_{1} \rightarrow \mathcal{G}_{2}$ is a horizontal group scheme homomorphism. For any $(E, \nabla) \in \operatorname{LocSys}_{\mathcal{G}_{1}}$ we can form the usual induced $\mathcal{G}_{2}$-torsor $h_{*} E=E \times^{\mathcal{G}_{1}, h} \mathcal{G}_{2}$. Moreover, there is a canonical isomorphism $p_{1}^{*} h_{*} E \simeq p_{2}^{*} h_{*} E$ on $\Delta^{(1)}$ which defines a connection $h_{*} \nabla$ on $h_{*} E$. Thus the assignment $(E, \nabla) \rightarrow\left(h_{*} E, h_{*} \nabla\right)$ defines a functor

$$
h_{*}: \operatorname{LocSys}_{\mathcal{G}_{1}} \rightarrow \operatorname{LocSys}_{\mathcal{G}_{2}},
$$

sometimes called the induction functor.

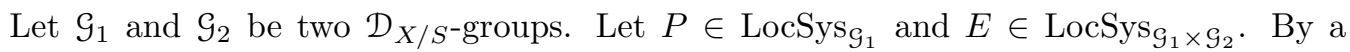
similar construction as above, we can form the induced $\mathcal{G}_{2}$-torsor

$$
P \otimes E:=P \times{ }^{\mathfrak{G}_{1}} E,
$$

equipped with a flat connection $\nabla_{P \otimes E}$. The assignment $\left(P, \nabla_{P}\right) \times\left(E, \nabla_{E}\right) \rightarrow\left(P \otimes E, \nabla_{P \otimes E}\right)$ defines a functor

$$
\otimes: \operatorname{LocSys}_{\mathcal{G}_{1}} \times \operatorname{LocSys}_{\mathcal{G}_{1} \times \mathcal{G}_{2}} \rightarrow \operatorname{LocSys}_{\mathcal{G}_{2}}
$$

sometimes called the tensor functor.

A.6. The $p$-curvature. Let us now assume that $p \mathcal{O}_{S}=0$. Let us first recall the notion of the $p$-curvature of a $\mathcal{D}_{X / S}$-module.

Let $\left(\mathcal{M}, \nabla: \mathcal{M} \rightarrow \mathcal{M} \otimes \Omega_{X / S}\right)$ be a $\mathcal{D}_{X / S}$-module. For any $v \in T_{X / S}$, let $v^{[p]} \in T_{X / S}$ be the $p$-th power of $v$. By [K, Proposition 5.2], the map

$$
T_{X / S} \rightarrow\left(F_{X}\right)_{*} \operatorname{End}_{\mathcal{O}_{X}}(\mathcal{M}), \quad v \rightarrow \nabla(v)^{p}-\nabla\left(v^{[p]}\right)
$$

is a morphism of $\mathcal{O}_{X}$-modules. By adjunction, we get a map

$$
\Psi(\nabla): F^{*} T_{X^{\prime} / S} \rightarrow \operatorname{End}_{\mathcal{O}_{X}}(\mathcal{M}),
$$

called the $p$-curvature of $(\mathcal{M}, \nabla)$.

Let $N \rightarrow X$ be an affine $\mathcal{D}_{X / S}$-scheme, we define the $p$-curvature of $N$ as the $p$-curvature $\Psi\left(\nabla_{N}\right)$ of the $\mathcal{D}_{X / S^{-}}$-algebra $\left(\mathcal{O}_{N}, \nabla_{N}\right)$. Using the formula of the $p$-curvature of a tensor connection $\Psi\left(\nabla_{1} \otimes \nabla_{2}\right)=\Psi\left(\nabla_{1}\right) \otimes$ id + id $\otimes \Psi\left(\nabla_{2}\right)$, we see that $\Psi\left(\nabla_{N}\right)$ factors through

$$
\Psi\left(\nabla_{N}\right): F^{*} T_{X^{\prime} / S} \rightarrow \operatorname{Der}_{\mathcal{O}_{X}}\left(\mathcal{O}_{N}\right) \subset \operatorname{End}_{\mathcal{O}_{X}}\left(\mathcal{O}_{N}\right) .
$$

Now let $\mathcal{G}$ be a smooth affine $\mathcal{D}_{X / S^{-}}$group scheme. It is easy to see that for any $v \in$ $F^{*} T_{X^{\prime} / S}$, the following diagram is commutative

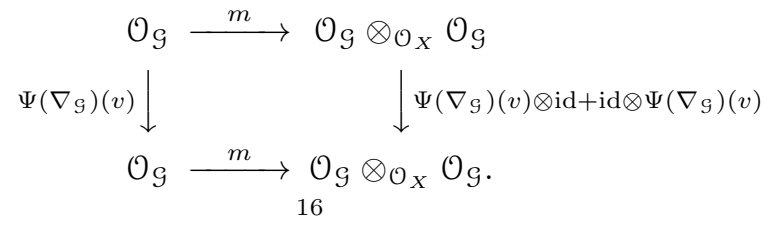


Likewise, let $(E, \nabla) \in$ LocSys $_{g}$. Then for any $v \in F^{*} T_{X^{\prime}}$, the following diagram is commutative

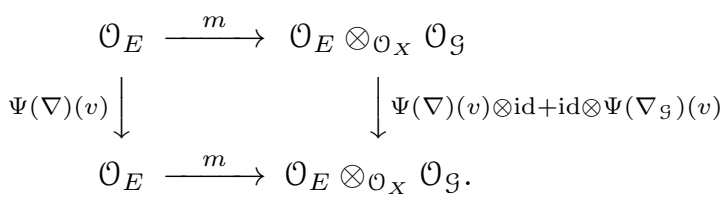

In particular, if $\Psi\left(\nabla_{\mathcal{G}}\right)=0$, then the image of $\Psi(\nabla)$ lands in $\operatorname{ad}(E) \subset \operatorname{Der}_{\mathcal{O}_{X}}\left(\mathcal{O}_{E}\right)$, and therefore the $p$-curvature mapping can be regarded as a section

$$
\Psi(\nabla) \in \operatorname{ad}(E) \otimes F^{*} \Omega_{X^{\prime} / S},
$$

which reduces to the standard notion of the $p$-curvature of a principal bundle with a flat connection (e.g. see [B, Appendix]).

A.7. The scheme of horizontal sections. To continue, we develop the theory of schemes of horizontal sections of a $\mathcal{D}_{X / S}$-scheme in characteristic $p$, analogous to [BD, Proposition 2.6.2].

Lemma A.3 (Cartier descent).

(1) For any quasi-coherent sheaf $\mathcal{L}$ on $X^{\prime}$, there is a canonical $\mathcal{D}_{X}$-module structure on $F^{*}(\mathcal{L})$ and the assignment $\mathcal{L} \rightarrow F^{*}(\mathcal{L})$ defines an equivalence between the category of quasi-coherent sheaves on $X^{\prime}$ and the category of $\mathcal{D}_{X}$-modules on $X$ with zero $p$-curvature, with an inverse functor given by taking flat sections $\mathcal{F} \mapsto \mathcal{F}^{\nabla}$.

(2) The above equivalence is a tensor equivalence, i.e., for any $\mathcal{L}_{1}, \mathcal{L}_{2} \in \mathrm{QCoh}\left(X^{\prime}\right)$ the natural isomorphism of $\mathcal{O}_{X^{\prime}}$-modules

$$
m: F^{*}(\mathcal{L}) \otimes F^{*}\left(\mathcal{L}_{2}\right) \simeq F^{*}\left(\mathcal{L}_{1} \otimes \mathcal{L}_{2}\right)
$$

is compatible with their $\mathcal{D}_{X}$-modules structures coming from part (1).

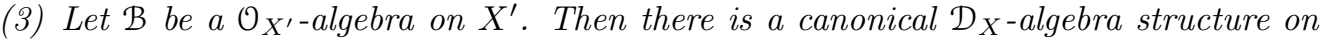
$F^{*}(\mathcal{B})$ and the assignment $\mathcal{L} \rightarrow F^{*}(\mathcal{L})$ defines an equivalence between the category of $\mathcal{O}_{X^{\prime}}$-algebras on $X^{\prime}$ and the category of $\mathcal{D}_{X^{-}}$-algebra on $X$ with zero $p$-curvature.

Proof. Part (1) is the standard Cartier descent. Part (3) follows from Part (2), which can be proved by a direct computation.

\section{Proposition A.4.}

(1) The functor $\mathcal{M}^{\prime} \rightarrow F^{*} \mathcal{M}^{\prime}$ admits a left adjoint functor, i.e. for a $\mathcal{D}_{X}$-algebra $\mathcal{N}$ there is a $\mathcal{O}_{X^{\prime}}$-algebra $H_{\nabla}(\mathcal{N})$ such that

$$
\operatorname{Hom}_{\mathcal{D}_{X} \text {-alg }}\left(\mathcal{N}, F^{*} \mathcal{M}^{\prime}\right)=\operatorname{Hom}_{\mathcal{O}_{X^{\prime}}-\operatorname{alg}}\left(H_{\nabla}(\mathcal{N}), \mathcal{M}^{\prime}\right)
$$

for any $\mathcal{O}_{X^{\prime}}$-algebra $\mathcal{M}^{\prime}$.

(2) The canonical map $\mathcal{N} \rightarrow F^{*}\left(H_{\nabla}(\mathcal{N})\right)$ is surjective.

Proof. Let $\nabla$ be the corresponding connection of $\mathcal{N}$. We denote by $\Psi$ the $p$-curvature of $\nabla$. We can think of $\Psi$ as a map $\Psi: F^{*} T_{X^{\prime}} \otimes_{\mathcal{O}_{X}} \mathcal{N} \rightarrow \mathcal{N}$. Let $\Psi(\mathcal{N})$ be the ideal of $\mathcal{N}$ generated by the image of $\Psi$ and define $\mathcal{N}_{\Psi}=\mathcal{N} / \Psi(\mathcal{N})$. Since $\nabla$ commutes with $\Psi$, the $\mathcal{O}_{X}$-algebra $\mathcal{N}_{\Psi}$ carries a connection and we define the following $\mathcal{O}_{X^{\prime}}$-algebra

$$
H_{\nabla}(\mathcal{N})=\left(\mathcal{N}_{\Psi}\right)^{\nabla}
$$

Let us show that $H_{\nabla}(\mathcal{N})$ satisfies our requirement. For any $\mathcal{O}_{X^{\prime}}$-algebra $\mathcal{N}^{\prime}$, the $p$-curvature of $F^{*} \mathcal{M}^{\prime}$ is zero, and therefore we have

$$
\operatorname{Hom}_{\mathcal{D}_{X} \text {-alg }}\left(\mathcal{N}, F^{*} \mathcal{M}^{\prime}\right)=\operatorname{Hom}_{\mathcal{D}_{X} \text {-alg }}\left(\mathcal{N}_{\Psi}, F^{*} \mathcal{M}^{\prime}\right)
$$

On the other hand, since the $p$-curvature of $\mathcal{N}_{\Psi}$ is zero, by Lemma A.3, we have

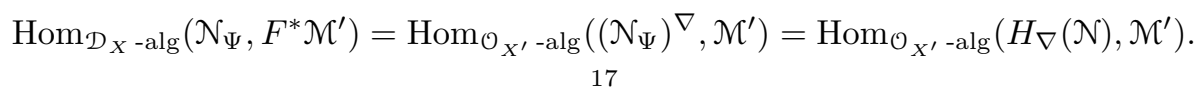


Therefore

$$
\operatorname{Hom}_{\mathcal{D}_{X}-\operatorname{alg}}\left(\mathcal{N}, F^{*} \mathcal{M}^{\prime}\right)=\operatorname{Hom}_{\mathcal{O}_{X^{\prime}}-\operatorname{alg}}\left(H_{\nabla}(\mathcal{N}), \mathcal{M}^{\prime}\right)
$$

This proved part 1).

By Lemma A.3 again, we have $\mathcal{N}_{\Psi}=F^{*}\left(H_{\nabla}(\mathcal{N})\right)$ and it implies the canonical map $\mathcal{N} \rightarrow \mathcal{N}_{\psi}=F^{*}\left(H_{\nabla}(\mathcal{N})\right)$ is surjective. This proved part 2).

Let $\mathcal{N}$ be a commutative $\mathcal{D}_{X}$-algebra. Then the $\mathcal{O}_{X^{\prime}}$-algebra $H_{\nabla}(\mathcal{N})$ is commutative by (2). We called the $X^{\prime}$-scheme

$$
N^{\nabla}:=\operatorname{Spec}\left(H_{\nabla}(\mathcal{N})\right)
$$

the scheme of horizontal sections. Let $N$ and $N^{\Psi}$ be the $\mathcal{D}_{X}$-scheme associated to the commutative $\mathcal{D}_{X}$-algebras $\mathcal{N}$ and $\mathcal{N}_{\Psi}$. We have

$$
N^{\Psi}=X \times \times_{X^{\prime}} N^{\nabla}, \quad\left(N^{\Psi}\right)^{\nabla}=N^{\nabla},
$$

and $N^{\Psi} \subset N$ is the maximal closed subscheme of $N$ that is constant with respect to the connection.

Example A.5. Let $\mathcal{G}^{\prime}$ be a smooth affine group scheme over $X^{\prime}$. Then by the theory of Cartier decent, the group scheme $\mathcal{G}:=F^{*} \mathcal{G}^{\prime}$ has a canonical connection, and for any $\mathcal{G}^{\prime}$ torsor $E^{\prime}$, there is a canonical connection on the $F^{*} \mathcal{G}^{\prime}$-torsor $F^{*} E^{\prime}$. One can easily check that the $p$-curvature of this connection is zero. Therefore, we have a functor

$$
F^{*}: \text { Bun }_{\mathcal{G}^{\prime}} \rightarrow \text { LocSys }_{\mathcal{G}}, \quad E^{\prime} \rightarrow F^{*} E^{\prime} .
$$

The functor $F^{*}$ induces an equivalence between the category of $\mathcal{G}^{\prime}$-torsors on $X^{\prime}$ and the category of $\mathcal{G}$-local systems with zero $p$-curvature. The inverse is given by $E \mapsto E^{\nabla}$.

A.8. The commutative case. Assume that $\mathcal{G}^{\prime}$ is commutative on $X^{\prime}$ and $\mathcal{G}=F^{*} \mathcal{G}^{\prime}$. For any $\mathcal{G}$-torsor $E, \operatorname{ad}(E) \simeq$ Lie $\mathcal{G}$ canonically. If $(E, \nabla) \in \operatorname{LocSys}_{\mathcal{G}}$, then by the Cartier descent, $\Psi(\nabla) \in \Gamma\left(X, \operatorname{Lie} \mathcal{G} \otimes F^{*} \Omega_{X^{\prime} / S}\right)$ is the pullback of a unique element in $\Gamma\left(X^{\prime}, \operatorname{Lie}^{\prime} \otimes \Omega_{X^{\prime} / S}\right)$. Therefore, taking $p$-curvature can be regarded as a map

$$
h_{p}: \operatorname{LocSys}_{\mathcal{G}} \rightarrow \Gamma\left(X^{\prime}, \operatorname{LieG}^{\prime} \otimes \Omega_{X^{\prime} / S}\right),
$$

where $T=\Gamma\left(X^{\prime}\right.$, LieG $\left.^{\prime} \otimes \Omega_{X^{\prime} / S}\right)$ is regarded as a space over $S$, whose fiber over $s \in S$ is $\Gamma\left(X_{s}^{\prime}\right.$, Lie $\left.\left.^{\prime}\right|_{X_{s}^{\prime}} \otimes \Omega_{X_{s}^{\prime}}\right)$. This is called the $p$-Hitchin map (see Theorem 3.1 for the noncommutative analogue).

As $\mathcal{G}$ is commutative, given two $\mathcal{G}$-local systems $\left(E_{1}, \nabla_{1}\right),\left(E_{2}, \nabla_{2}\right)$, the induction $E_{1} \times{ }^{\mathcal{G}} E_{2}$ is a natural G-local system. Then we have

$$
h_{p}\left(E_{1} \times{ }^{\mathcal{G}} E_{2}\right)=h_{p}\left(E_{1}\right)+h_{p}\left(E_{2}\right) .
$$

Combining with Example A.5, we have

Corollary A.6. Assume that $\mathcal{G}^{\prime}$ is commutative. Then the stack of $\mathcal{G}$-local systems with a fixed $p$-curvature $\psi \in T$ is a (pseudo) torsor under the Picard stack Bun $\mathcal{G}^{\prime}$.

We will give an interpretation of this (pseudo) Bun $\mathcal{G}^{\prime}$-torsor, generalizing [OV, Proposition 4.13] for $\mathcal{G}=\mathbb{G}_{m}$.

Let $z_{X / S}$ be the sheaf of closed one forms on $X$. Then $F_{*} z_{X / S}$ is an $\mathcal{O}_{X^{\prime}}$-module. Recall the definition of the sheaf of flat connections $\left(\operatorname{Lie} \mathcal{G} \otimes \Omega_{X / S}\right)^{c l}$ on the trivial $\mathcal{G}$-torsor $E^{0}$ as in $\S$ A.3. Under our assumptions of $\mathcal{G}, F_{*}\left(\operatorname{Lie} \mathcal{G} \otimes \Omega_{X / S}\right)^{c l}=\mathrm{Lie}^{\prime} \otimes_{\mathcal{O}_{X^{\prime}}} F_{*} z_{X / S}$, where $z_{X / S}$ is the sheaf of closed one-forms on $X$. Then the $p$-Hitchin map $h_{p}$ induces an additive map of étale sheaves on $X^{\prime}$,

$$
h_{p}: \operatorname{Lie}^{\prime} \otimes F_{*} z_{X / S} \rightarrow \operatorname{Lie}^{\prime} \otimes \Omega_{X^{\prime} / S} .
$$

As in the case $\mathcal{G}=\mathbb{G}_{m}$, this map fits into the following four term exact sequence of sheaves on $X_{e t}^{\prime}$. 
Proposition A.7. Over $X_{e t}^{\prime}$, there is the exact sequence of étale sheaves

$$
1 \longrightarrow \mathcal{G}^{\prime} \longrightarrow F_{*} \mathcal{G} \stackrel{F_{*} d \log }{\longrightarrow} \mathrm{Lie}^{\prime} \otimes F_{*} z_{X / S} \stackrel{h_{p}}{\longrightarrow} \mathrm{Lie}^{\prime} \otimes \Omega_{X^{\prime} / S} \longrightarrow 1,
$$

where $d \log$ is defined as in $\S A .3$.

Proof. It is clear from the definition of $d \log$ that the kernel of $F_{*} d \log$ is $\mathcal{G}^{\nabla}=\mathcal{G}^{\prime}$. Next, we show that the sequence is exact at $\operatorname{Lie}^{\prime} \otimes F_{*} z_{X / S}$. As explained in $\S A .3,\left(E^{0}, \nabla^{0}+d \log (g)\right) \simeq$ $\left(E^{0}, \nabla^{0}\right)$. As the latter has zero $p$-curvature, we see that $h_{p} \circ F_{*} d \log =0$. On the other hand, if $\nabla=\nabla^{0}+\omega$ is a flat connection on $E^{0}$ with zero $p$-curvature, then by Example A.5 $E^{\prime}=\left(E^{0}\right)^{\nabla}$ is a $\mathcal{G}^{\prime}$-torsor. and there is a canonical isomorphism $\alpha: F^{*} E^{\prime} \simeq E^{0}$. Étale locally, we can trivialize $E^{\prime}$ so we can choose $\beta:\left(E^{0}\right)^{\prime} \simeq E^{\prime}$. Then we obtain a section $g=\alpha \circ F^{*} \beta \in \mathcal{G}$, and it is not hard to check that $\omega=d \log (g)$. Note that if $\mathcal{G}=\mathbb{G}_{m}$, the argument shows that this sequence is exact at Lie $\mathcal{G}^{\prime} \otimes F_{*} z_{X / S}$ even Zariski locally on $X^{\prime}$, which is well-known.

Finally, we show that $h_{p}$ is surjective. Unlike $\mathcal{G}=\mathbb{G}_{m}$, there is no explicit formula for the $p$-linear map of LieG, and the usual argument by explicit calculations does not apply here.

Recall that if $\mathcal{V}$ is a locally free $\mathcal{O}_{X^{\prime}}$-module of finite rank, $\operatorname{Spec}_{\operatorname{Sym}} \mathcal{O}_{X^{\prime}} \mathcal{V}^{\vee}$ is a vector bundle on $X^{\prime}$ whose sheaf of sections are $\mathcal{V}$. Let $\mathbb{V}_{1}$ and $\mathbb{V}_{2}$ be the vector bundles on $X^{\prime}$ corresponding to LieG $\mathcal{G}^{\prime} \otimes F_{*} z_{X / S}$ and LieG $\mathcal{G}^{\prime} \otimes \Omega_{X^{\prime} / S}$ respectively. Although $h_{p}$ is not $\mathcal{O}_{X^{\prime}}$-linear, we have

Lemma A.8. The map $h_{p}$ is induced by a smooth surjective homomorphism of commutative group schemes $\mathbb{h}_{p}: \mathbb{V}_{1} \rightarrow \mathbb{V}_{2}$.

Assuming the lemma, the surjectivity of $h_{p}$ then is clear. Namely, let $s: X^{\prime} \rightarrow \mathbb{V}_{2}$ be a section, then étale locally on $X^{\prime}, s$ can be lifted to a section of $\mathbb{V}_{1}$ as $\mathbb{h}_{p}$ is smooth surjective.

It remains to prove the lemma. We use an argument similar to [OV, Proposition 2.5 (1)]. We first give another description of the map $h_{p}$. Let $\mathcal{C}_{X / S}: F_{*} z_{X / S} \rightarrow \Omega_{X^{\prime} / S}$ be the Cartier operator. Let $\pi_{X / S}: X^{\prime} \rightarrow X$ is the map over the absolute Frobenius $F r_{S}$ of $S$. Let $(-)^{p}: \mathrm{Lie}^{\prime} \rightarrow \mathrm{LieG}^{\prime}$ be the map given by the $p$-Lie algebra structure of Lie $\mathcal{G}^{\prime}$. We claim that for $z \otimes \omega \in \operatorname{Lie} \mathcal{G}^{\prime} \otimes F_{*} z_{X / S}$,

$$
h_{p}(z \otimes \omega)=z^{p} \otimes \pi_{X / S}^{*}(\omega)-z \otimes \mathcal{C}_{X / S}(\omega) .
$$

Indeed, let $\nabla^{0}$ be the canonical connection on the trivial $\mathcal{G}$-torsor $E^{0}$. Then for a vector field $\xi \in T_{X / S}$, and $z \otimes \omega \in \mathrm{LieG}^{\prime} \otimes F_{*} z_{X / S}$, one has (as sections of $\widetilde{T}_{E^{0}}$ )

$$
\left(\nabla_{\xi}^{0}+(\omega, \xi) z\right)^{p}-\left(\left(\nabla_{\xi}^{0}\right)^{p}+\left(\omega, \xi^{p}\right) z\right)=\xi^{p-1}(\omega, \xi) z+(\omega, \xi)^{p} z^{p}-\left(\omega, \xi^{p}\right) z,
$$

by the Jacobson identity. By the definition of the Cartier operator, $\left(\omega, \xi^{p}\right)-\xi^{p-1}(\omega, \xi)=$ $\left(\mathcal{C}_{X / S}(\omega), \pi_{X / S}^{*}(\xi)\right)$. The claim follows.

We apply (A.8) as follows: The Cartier operator $\mathcal{C}_{X / S}$ is $\mathcal{O}_{X^{\prime}}$-linear and surjective, and therefore is induced by a smooth homomorphism $\mathbb{C}_{X / S}: \mathbb{V}_{1} \rightarrow \mathbb{V}_{2}$ of the underlying commutative group schemes. The map $z \otimes \omega \mapsto z^{p} \otimes \pi_{X / S}^{*}(\omega)$ is not $\mathcal{O}_{X^{\prime}}$-linear, but is still induced from an inseparable homomorphism $\Phi: \mathbb{V}_{1} \rightarrow \mathbb{V}_{2}$ of the commutative group schemes. In fact, let $F r_{X^{\prime}}: X^{\prime} \rightarrow X^{\prime}$ be the absolute Frobenius of $X^{\prime}$. We have $\mathcal{O}_{X^{\prime}}$-linear maps $F r_{X^{\prime}}^{*}$ Lie $\mathcal{G}^{\prime} \rightarrow$ LieG' given by the $p$-Lie algebra structure and $F r_{X^{\prime}}^{*} F_{*} z_{X / S} \rightarrow \Omega_{X^{\prime} / S}$ given by the composition $F r_{X^{\prime}}^{*} F_{*} z_{X / S} \rightarrow F r_{X^{\prime}}^{*} F_{*} \Omega_{X / S} \rightarrow \Omega_{X^{\prime} / S}$. Therefore, we have a commutative group scheme homomorphism $\mathbb{V}_{1}^{\left(X^{\prime}\right)} \rightarrow \mathbb{V}_{2}$. It is then readily to see that $\Phi$ is the composition of the relative Frobenius $\mathbb{V}_{1} \rightarrow \mathbb{V}_{1}^{\left(X^{\prime}\right)}$ (which is a group homomorphism) with the above homomorphism.

Now $\mathbb{h}_{p}=\Phi-\mathbb{C}_{X / S}$ is a smooth morphism, as $\Phi$ is inseparable and the differential of $\mathbb{C}_{X / S}$ is surjective. It remains to show that $\Phi-\mathbb{C}_{X / S}$ is surjective at the level of points. We can base change $\mathbb{h}_{p}$ to a geometric point of $x \in X^{\prime}$. As $\mathbb{h}_{p}$ is smooth, the image contains 
an open subgroup of $\left(\mathbb{V}_{2}\right)_{x}$, as $\left(\mathbb{V}_{2}\right)_{x}$ is connected, this subgroup must be the entire $\left(\mathbb{V}_{2}\right)_{x}$. The lemma follows.

Now, we will give an interpretation of the pseudo Bun $\mathcal{G}^{\prime}$-torsor in Corollary A.6. Namely, the $p$-curvature $\psi$ gives rise to a section of $\mathrm{LieG}^{\prime} \otimes \Omega_{X^{\prime} / S}$, and the 4 -term exact sequence A.7 induces a $\mathcal{G}^{\prime}$-gerbe $\mathscr{G}_{\psi}$ on $X^{\prime}$.

Proposition A.9. Then the pseudo Bun $\mathcal{G}^{\prime}$-torsor as in Corollary A.6 is the stack of splittings of the gerbe $\mathscr{G}_{\psi}$ over $S$.

The proof is similar to $[\mathrm{OV}, \S 4$, Proposition 4.2]. One only needs to replace the invertible sheaf $L$ associated to the $F_{Y / S *}\left(\mathcal{O}_{Y}^{*}\right)$-torsor $\mathcal{L}$ in loc. cit. by the structure sheaf $\mathcal{O}_{E}$ of the $F_{*} \mathcal{G}$-torsor $E$ in the current setting.

A.9. In this subsection we assume that $S=$ Speck, where $k$ algebraically closed of characteristic $p>0$. We describe the tangent map of $h_{p}: \operatorname{LocSys}_{\mathcal{G}} \rightarrow \Gamma\left(X^{\prime}, \operatorname{Lie}^{\prime} \otimes \Omega_{X^{\prime} / k}\right)$ at the trivial $\mathcal{G}$-torsor with the canonical connection $x=\left(E^{0}, \nabla^{0}\right)$. First, the tangent space of $\operatorname{LocSys}_{\mathcal{G}}$ at $x$ is given by hypercohomology $\mathbb{H}^{1}$ of the following deRham complex

$$
\Omega_{X / k}^{\bullet}(\operatorname{Lie} \mathcal{G}):=\left\{0 \rightarrow \operatorname{Lie} \mathcal{\nabla ^ { 0 }} \rightarrow \operatorname{Lie} \mathcal{G} \otimes \Omega_{X / k}^{1} \rightarrow \operatorname{Lie} \mathcal{G} \otimes \Omega_{X / k}^{2} \rightarrow \cdots\right\} .
$$

Recall that there is the "second spectral sequence" with $E^{i}=\mathbb{H}^{i}\left(X, \Omega_{X / k}^{\bullet}(\operatorname{Lie} \mathcal{G})\right)$ and $E_{2}^{i, j}=H^{i}\left(X, \mathcal{H}^{j}\left(\Omega_{X / k}^{\bullet}(\operatorname{Lie} \mathcal{G})\right)\right)$. When $\mathcal{G}=\mathbb{G}_{m}$, this is also known as the conjugate spectral sequence. In particular

$$
E_{2}^{0,1}=\Gamma\left(X, \mathcal{H}^{1}\left(\Omega_{X / k}^{\bullet}(\operatorname{Lie} \mathcal{G})\right) \simeq \operatorname{Lie}^{\prime} \otimes \Omega_{X^{\prime} / k} .\right.
$$

Here the last isomorphism is obtained by the Lie algebra version of the exact sequence as in Proposition A.7. Note that if $\mathcal{G}=\mathbb{G}_{m}$ or $\mathbb{G}_{a}$, it is just the usual Cartier isomorphism. The edge morphism of the spectral sequence induces

$$
c: T_{x} \operatorname{LocSys}_{\mathcal{G}} \simeq \mathbb{H}^{1}\left(X, \Omega_{X / k}^{\bullet}(\mathrm{Lie} \mathcal{G})\right) \rightarrow H^{0}\left(X^{\prime}, \operatorname{Lie}^{\prime} \otimes \Omega_{X^{\prime} / k}^{1}\right) .
$$

Lemma A.10. The map $c$ is equal to $-d h_{p}$.

Proof. We follow the argument in [OV, Lemma 4.12]. Recall that we have a natural inclusion $i: H^{0}\left(X^{\prime}, \operatorname{Lie}^{\prime} \otimes F_{*} z_{X / k}^{1}\right) \rightarrow$ LocSys $_{\mathcal{G}}$ of the substack of flat connections on the trivial torsor $E^{0}$. It follows by definition that

$$
-d\left(h_{p} \circ i\right)=c \circ d i: H^{0}\left(X, \operatorname{Lie}^{\prime} \otimes F_{*} z_{X / k}^{1}\right) \rightarrow H^{0}\left(X^{\prime}, \operatorname{Lie}^{\prime} \otimes \Omega_{X^{\prime} / k}^{1}\right) .
$$

More explicitly, they are equal to the Cartier map $\mathcal{C}_{X / S}$ as introduced in the proof of Proposition A.7. Now let $v \in \mathbb{H}^{1}\left(X, \Omega_{X / k}^{\bullet}(\operatorname{Lie} \mathcal{G})\right)$ and let $(E, \nabla)$ be the corresponding $\mathcal{G}^{-}$ torsor with connection on $X[\epsilon]$. The section $d h_{p}(v) \in H^{0}\left(X^{\prime}, \mathrm{Lie}^{\prime} \otimes \Omega_{X^{\prime} / k}^{1}\right)$ is determined by its pull back to any étale cover of $X^{\prime}$. We can choose an étale cover on which $E$ is trivial, hence reduced to the case when $E$ is trivial and $v \in H^{0}\left(X, \operatorname{Lie}^{\prime} \otimes F_{*} Z_{X / k}^{1}\right)$.

\section{REFERENCES}

[BNR] A. Beauville, M.S. Narasimhan, S. Ramanan: Spectral curves and the generalised theta divisor, J. Reine Angew. Math. (1989) Volume: 398, page 169-179.

[BD] A. Beilinson, V. Drinfeld: Quantization of Hitchin's integrable system and Hecke eigensheaves, preprint, available at http://www.math.uchicago.edu/ mitya/langlands/.

[BB] R. Bezrukavnikov. A. Braverman.: Geometric Langlands conjecture in characteristic p: The $G L_{n}$ case, Pure Appl. Math. Q. 3 (2007), no. 1, Special Issue: In honor of Robert D. MacPherson. Part 3, 153-179.

[B] J.B. Bost: Algebraic leaves of algebraic foliations over number fields, Publ. Math. Inst. Hautes Étud. Sci. No. 93 (2001), 161-221.

[CGP] B. Conrad, O. Gabber, G. Prasad: Pseudo-reductive groups, New mathematical momographs: 17.

[CZ] T.H. Chen, X. Zhu: Geometric Langlands in prime characteristic, arXiv:1403.3981. 
[DG] R. Donagi, D. Gaitsgory: The gerbe of Higgs bundles, Transformation groups Volume 7, Number 2, 109-153

[G] M. Groechenig: Moduli of flat connections in positive characteristic, arXiv:1201.0741.

[I] L. Illusie: Complexe de deRham-Witt et cohomologie cristalline, Ann. Sci. École Norm. Sup. (4) 12 (1979), no. 4, 501-661.

[K] N. Katz: Nilpotent connections and the Monodromy Theorem, Publ. Math. Inst. Hautes Étud. Sci. No. 39 (1970), 175-232.

[LP] Y. Laszlo, C. Pauly: On the Hitchin morphism in positive characteristic, Internat. Math. Res. Notices 2001, no. 3, 129-143.

[MM] B. Mazur, W. Messing: Universal extensions and one dimensional crystalline cohomology, Lecture Notes in Mathematics 370.

[N1] B.C. Ngô: Hitchin fibration and endoscopy, Invent. Math. 164 (2006) 399-453.

[N2] B.C. Ngô: Le lemme fondamental pour les algèbres de Lie, Publ. Math. Inst. Hautes Étud. Sci. No. 111 (2010), 1-169.

[O] A. Ogus: Higgs cohomology, p-curvature, and the Cartier isomorphism, Compositio Math. 140 (2004) $145-164$.

[OV] A. Ogus, V. Vologodsky: Nonabelian Hodge theory in characteristic p, Publ. Math. Inst. Hautes Étud. Sci. No. 106 (2007), pp.1-138

[S] C. Simpson: Higgs bundles and local systems, Publ. Math. Inst. Hautes Étud. Sci. No. 75 (1992), 5-95.

Department of Mathematics, Northwestern University, 2033 Sheridan Road, Evanston, IL 60208, USA

E-mail address: chenth@math.northwestern.edu

Department of Mathematics, California Institute of Technology, 1200 E. California Blvd., Pasadena, CA 91125, USA

E-mail address: xzhu@caltech.edu 